\title{
Complement receptor C3aR1 controls neutrophil mobilization following spinal cord injury through physiological antagonism of CXCR2
}

Faith H. Brennan, ${ }^{1}$ Trisha Jogia,, ${ }^{1}$ Ellen R. Gillespie, ${ }^{1}$ Linda V. Blomster, ${ }^{1}$ Xaria X. Li, ${ }^{1}$ Bianca Nowlan, ${ }^{2}$ Gail M. Williams, ${ }^{3}$ Esther Jacobson, ${ }^{1}$ Geoff W. Osborne, ${ }^{4}$ Frederic A. Meunier, ${ }^{4}$ Stephen M. Taylor, ${ }^{1}$ Kate E. Campbell,, ${ }^{5,6}$ Kelli P.A. MacDonald, ${ }^{7}$ Jean-Pierre Levesque, ${ }^{2}$ Trent M. Woodruff, ${ }^{1}$ and Marc J. Ruitenberg ${ }^{1,8}$

${ }^{1}$ School of Biomedical Sciences, Faculty of Medicine, ${ }^{2}$ Blood and Bone Diseases Program, Mater Research Institute, ${ }^{3}$ School of Public Health, Faculty of Medicine, and ${ }^{4}$ Clem Jones Centre for Ageing Dementia Research, Queensland Brain Institute, The University of Queensland, Brisbane, Australia. ${ }^{5}$ Orthopaedic Department, Princess Alexandra Hospital, Brisbane, Australia. ${ }^{6}$ Princess Alexandra Hospital - Southside Clinical School, Faculty of Medicine, The University of Queensland, Brisbane, Australia. ${ }^{7}$ Antigen Presentation and Immunoregulation Laboratory, QIMR Berghofer Medical Research Institute, Brisbane Australia. ${ }^{8}$ Trauma, Critical Care and Recovery, Brisbane Diamantina Health Partners, Brisbane, Australia.

Traumatic spinal cord injury (SCI) triggers an acute-phase response that leads to systemic inflammation and rapid mobilization of bone marrow (BM) neutrophils into the blood. These mobilized neutrophils then accumulate in visceral organs and the injured spinal cord where they cause inflammatory tissue damage. The receptor for complement activation product $3 a$, C3aR1, has been implicated in negatively regulating the BM neutrophil response to tissue injury. However, the mechanism via which C3aR1 controls BM neutrophil mobilization, and also its influence over SCI outcomes, are unknown. Here, we show that the $\mathrm{C3a} / \mathrm{C} 3 \mathrm{aR} 1$ axis exerts neuroprotection in $\mathrm{SCI}$ by acting as a physiological antagonist against neutrophil chemotactic signals. We show that C3aR1 engages phosphatase and tensin homolog (PTEN), a negative regulator of the phosphatidylinositol 3-kinase (PI3K)/AKT pathway, to restrain C-X-C chemokine receptor type 2-driven BM neutrophil mobilization following trauma. These findings are of direct clinical significance as lower circulating neutrophil numbers at presentation were identified as a marker for improved recovery in human SCI. Our work thus identifies C3aR1 and its downstream intermediary, PTEN, as therapeutic targets to broadly inhibit neutrophil mobilization/recruitment following tissue injury and reduce inflammatory pathology.

Conflict of interest: FHB, SMT, TMW, and MJR are named as Contributor or Inventor on patent PCT/ AU2016/050435 regarding the use of C3aR1-targeted drugs in inflammatory conditions

Copyright: (c) 2019 American Society for Clinical Investigation

Submitted: May 23, 2018 Accepted: March 21, 2019 Published: May 2, 2019.

Reference information: /CI Insight. 2019;4(9):e98254. https://doi. org/10.1172/jii.insight.98254.

\section{Introduction}

The bone marrow (BM) stores a large pool of mature neutrophils that is rapidly mobilized in response to injury and/or infection. Egress of these cells is critical for any successful immune response, but uncontrolled neutrophil accumulation and activity in inflamed tissues can also drive pathology $(1,2)$. The mobilization of BM neutrophils is therefore tightly regulated by a complex interplay of cytokines, chemokines, pattern recognition receptors, and cell adhesion molecules (3). Our previous work indicated an unexpected negative regulatory role for the G protein-coupled receptor (GPCR) of complement activation product $3 \mathrm{a}, \mathrm{C} 3 \mathrm{aR} 1$, in this process (4). How C3aR1 controls the egress of BM neutrophils remained to be elucidated.

Activation of C3aR1 by its natural ligand, C3a, a 77-amino acid cleavage product of C3 proteolysis, was traditionally viewed as predominantly proinflammatory (5). Context-specific immunoregulatory roles for $\mathrm{C} 3 \mathrm{a} / \mathrm{C} 3 \mathrm{aR} 1$ have, however, emerged over the last decade, and they include the homing and/or retention of hematopoietic stem/progenitor cells $(6,7)$ as well as neutrophils (4) in the BM. Understanding how these $\mathrm{C} 3 \mathrm{a} / \mathrm{C} 3 \mathrm{aR} 1$ effects are mediated is key to the development of novel therapeutic approaches to attenuate inflammation, particularly in neutrophil-driven pathologies. 
Neutrophil infiltration after insult is a prominent feature of both human and experimental traumatic spinal cord injury (SCI) (8-10), and presence of these cells at the lesion site is thought to contribute considerably to secondary inflammatory pathology and thus worse outcomes (11-14). Relatively little is known, however, about the molecular mechanisms orchestrating neutrophil mobilization and recruitment to the injured spinal cord, and to the best of our knowledge no study to date has explored a role for C3aR1 in this pathology.

Here, we first delineated a role for $\mathrm{C} 3 \mathrm{a} / \mathrm{C} 3 \mathrm{aR} 1$ in SCI by comparing the recovery of wild-type (WT) and $\mathrm{C}_{3} \mathrm{ar}^{{ }^{-1-}}$ (i.e., knockout) mice from blunt spinal cord trauma, one of the most common types of SCI in humans (15). Because C3aR1 is expressed by cells of myeloid $(16,17)$ and central nervous system (CNS) origin $(18,19)$, we also used BM chimera approaches to disentangle peripheral from central C3a/C3aR1 roles in relation to SCI outcomes. We then employed a variety of genetic and pharmacological approaches, including in vitro and in vivo functional assays, antibody-mediated neutrophil depletion, and chemotaxis assays to demonstrate that $\mathrm{C} 3 \mathrm{aR} 1$ engages phosphatase and tensin homolog (PTEN) to negatively regulate granulocyte egress from the BM into the circulation in response to inflammatory stimuli. These findings are significant from a therapeutic perspective, as a greater number of circulating neutrophils in the blood was associated with worse outcomes in both mouse and human SCI.

\section{Results}

SCI leads to C3a generation, leukocyte infiltration, and elevated C3aR1 expression. To begin exploring a role for $\mathrm{C} 3 \mathrm{a}$ in SCI, we first assessed the time course of its generation. C3a levels in the mouse spinal cord rapidly increased following injury (Figure 1A), and they were significantly elevated over sham-operated controls at 6,12 , and 24 hours after surgery ( $>5$-fold increase; $P<0.001$ ). Plasma C3a levels also rose sharply within 30 minutes of SCI, and remained elevated over sham-operated controls for at least 1 day after SCI (Figure 1B). Select key comparisons of $\mathrm{C} 3 \mathrm{a}$ levels in plasma and spinal cord samples of $\mathrm{C}_{3}$ ar1 ${ }^{-/-}$mice yielded similar results, suggesting a similar magnitude of complement system activation between genotypes (2 hours after surgery: $C 3$ arl $^{-/-}$plasma $7.93 \pm 1.63 \mu \mathrm{g} / \mathrm{ml}$ vs. WT plasma $6.94 \pm 0.90 \mu \mathrm{g} / \mathrm{ml}, n=4-5$ per genotype, $P=0.51$; $C 3 \mathrm{ar}^{{ }^{-/-}}$spinal cord $0.76 \pm 0.10 \mathrm{pg} / \mu \mathrm{g}$ vs. WT spinal cord $0.68 \pm 0.08 \mathrm{pg} / \mu \mathrm{g}, n=$ 4 per genotype, $P=0.58)$.

Widespread C3aR1 staining was observed at and around the site of SCI, and on a variety of cell types. In the acute phase, C3aR1-expressing Ly6B.2 $2^{+}$and $\mathrm{CD} 11 \mathrm{~b}^{+}$cells were abundant at and around the lesion site at 1 day after injury (Figure $1, C$ and $D$ ), a time point that coincides with peak neutrophil recruitment (20). The majority of infiltrating Ly6B.2 $2^{+}$cells were genuine neutrophils, as little overlap was observed between Ly6B.2 staining and $\mathrm{GFP}^{+}$cells of monocytic lineage in $\mathrm{C} x 3 \mathrm{cr} \mathrm{I}^{\mathrm{gp} / \mathrm{l}}$ mice at this time point (Figure 1E). Overall, these findings are consistent with C3aR1 being expressed by activated neutrophils $(16,21)$. At 35 days after SCI, when the lesion site is dominated by macrophages and a glial scar (22), C3aR1 expression remained high and was observed on a variety of cell types; these included $\mathrm{GFAP}^{+}$astrocytes, Iba $1^{+}$ microglia/macrophages, and at least a subset of residual Ly6B.2 $2^{+}$neutrophils (Figure 1, F-H).

Genetic ablation of C3aR1 worsens SCI outcomes and exacerbates inflammation. To determine whether C3a/ C3aR1 affects the neurological outcome from SCI, we next assessed the recovery of WT and C3ar $1^{-{ }^{--}}$mice from SCI. Over-ground locomotion was normal in both genotypes prior to surgery (time point 0 ), and SCI induced near-complete paralysis of the hindlimbs at 1 day after injury (Basso Mouse Scale [BMS] locomotor score [ref. 23]: 0-1; Figure 2A). Strikingly, C3ar1-/- mice regained significantly less locomotor function during the postacute phase of injury compared with their WT counterparts, as evident from lower BMS scores at 14, 21, 28, and 35 days after SCI $(P<0.05$; Figure 2, A and B). Consistent with worse functional outcomes, C3ar1 ${ }^{-/-}$mice had larger lesion volumes on $\mathrm{T}_{2}{ }^{*}$-weighted MRI (Figure 2, C and D; $P<0.01$ ). Myelin content at the lesion epicenter, which closely correlates with behavioral deficits/outcomes (24), was also significantly lower in the absence of $\mathrm{C} 3 \mathrm{aR} 1(P<0.01$; Figure $2, \mathrm{E}$ and $\mathrm{F})$. Assessment of astrogliosis revealed no differences between genotypes in either the $\mathrm{GFAP}^{+}$area (Figure 2, $\mathrm{E}$ and $\mathrm{G} ; P=0.18$ ) or the intensity of GFAP staining $(P=0.73)$ at 35 days after SCI. Nissl staining further revealed a significantly greater abnormal granular tissue presence $(P<0.01$; Figure $2, \mathrm{H}$ and $\mathrm{I})$, and an increase in the inflammatory infiltrate at the lesion epicenter of $C 3 a r 1^{-/-}$mice at 35 days after SCI $(P<0.05$; Supplemental Figure 1; supplemental material available online with this article; https://doi.org/10.1172/jci.insight.98254DS1).

Loss of C3aR1 leads to acute granulocytosis in response to trauma. Given the various indicators of worsened outcomes and increased post-SCI inflammation in $C 3$ ar1 $1^{-/-}$mice, we next assessed whether a loss of C3aR1 changed the nature and/or magnitude of the inflammatory response at the outset. Ly6B. $2^{+}$neutrophils, i.e., 
A

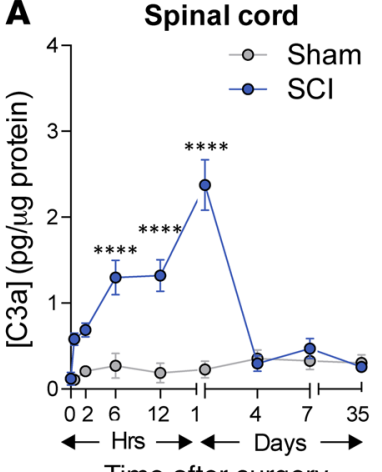

Time after surgery

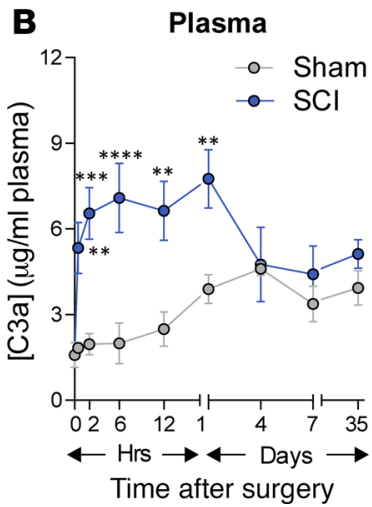

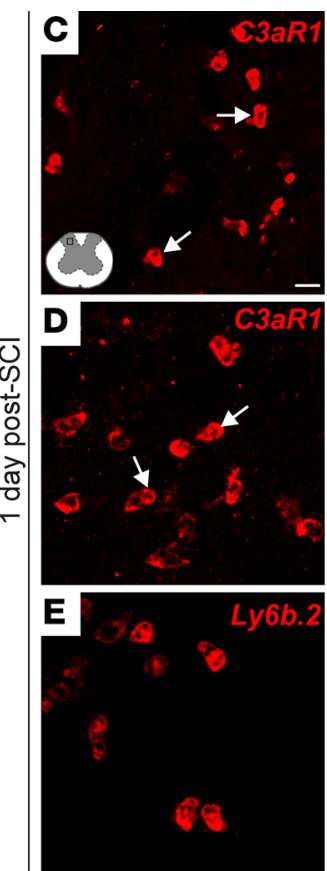
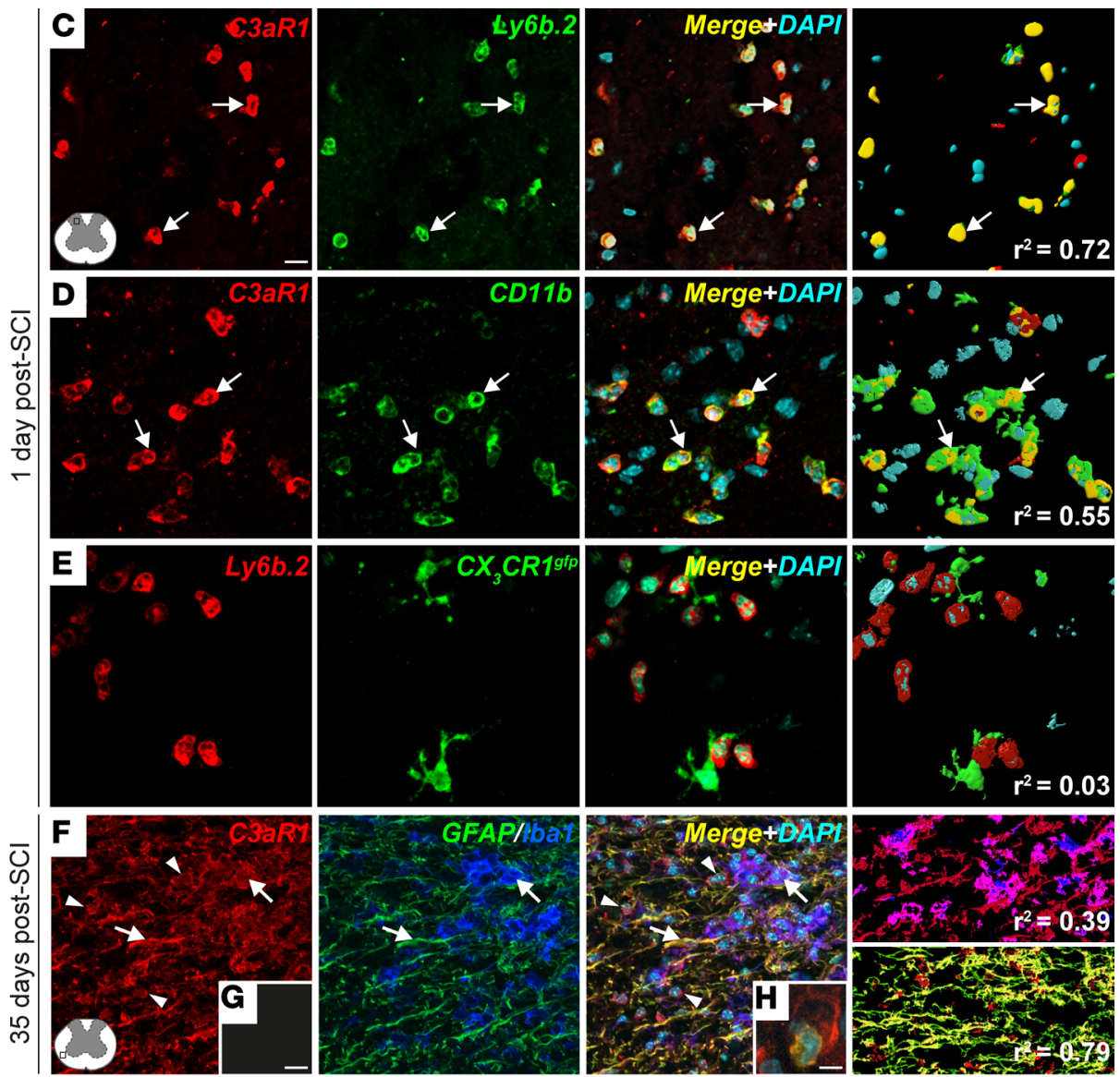

Figure 1. C3a production after $\mathrm{SCl}$ and expression of its receptor, C3aR1, in the damaged neural parenchyma. (A) C3a levels in the spinal cord rapidly increased in response to injury, peaking at 1 day after $\mathrm{SCl}$. (B) Circulating C3a levels were also significantly increased within 2 hours of SCl, and they remained well above those observed in sham-operated control mice for up to 1 day after injury. Data points are mean \pm SEM ( $n=4-5$ per genotype per time point). ${ }^{* *} P<0.01,{ }^{* *} P<0.001 ;{ }^{* * *} P<0.0001$ by 2 -way ANOVA with Bonferroni's post hoc test (SCI vs. time-matched sham-operated control). (C-F) Representative images of C3aR1 (or Ly6B.2 in E; red) staining during the acute (1 day) and more chronic (35 days) phase of SCl. Merged images of costains (green and/or blue) with nuclear dye (cyan), and Imaris surface reconstructions for colocalization analysis are shown on the right $\left(r^{2}\right.$ value $=$ square of Pearson's correlation for colocalization). C3aR1 was expressed on both Ly6B. $2^{+}$(C) and CD11b+ (D) cells. (E) Ly6B.2 $2^{+}$cells in the injured spinal cord of Cx3cr19fp/+ mice do not express GFP, indicating that they are infiltrating neutrophils. (F) Representative image showing C3aR1 staining in WT spinal cord at 35 days after injury. C3aR1 colocalized to amoeboid-shaped Iba1+ microglia/macrophages (blue) and fibrous GFAP+ astrocytes (green); other C3aR1-expressing cells can also be seen (arrowheads). (C) Confirmation of C3aR1 staining and antibody specificity on lesioned C3art ${ }^{-/-}$spinal cord tissue. (H) Higher-power confocal image of an infiltrating Ly6B. $2^{+}$neutrophil (green) coexpressing C3aR (red) in the spinal cord at 35 days after injury. Images are representative of 3 mice per time point and condition. Scale bars: $14 \mu \mathrm{m}$ (C), $20 \mu \mathrm{m}$ (C), and $4 \mu \mathrm{m}(\mathbf{H})$.

the first white blood cells (WBCs) to enter into the lesioned spinal cord, were indeed present in significantly greater numbers in $\mathrm{C}_{3} \mathrm{arl}^{-1-}$ mice (Figure 3A; $P<0.05$ ); this difference from WT mice was apparent as early as 1 day after injury and did not normalize during the postacute phase. Thus, absence of $\mathrm{C} 3 \mathrm{aR} 1$ augments the inflammatory response to SCI, beginning with an increased presence of Ly6B. $2^{+}$neutrophils.

We then explored if the greater presence of Ly6B.2 $2^{+}$cells at the site of SCI in C3ar1 $1^{-1-}$ mice resulted from increased BM neutrophil mobilization in response to trauma-induced tissue damage. WBC analysis indeed revealed that significantly more $\mathrm{Gr}-1^{+} \mathrm{SSC}^{\text {hi }}$ neutrophils were being mobilized into the blood of $C 3$ ar1 $1^{-/}$mice at 2 hours after SCI $\left(C 3 a r 1^{-1-} 1.6 \pm 0.17 \times 10^{6}\right.$ cells $/ \mathrm{ml}$ vs. WT $0.91 \pm 0.18$ $\times 10^{6} \mathrm{cells} / \mathrm{ml} ; P<0.01$; Figure $3 \mathrm{~B}$ ), with a concurrent reduction in the number of these cells at the level of the BM $(P<0.05$; Figure $3 \mathrm{C})$. Acute differences in SCI-induced BM neutrophil mobilization between genotypes could not be explained based on lesion severity (see Methods) and/or the degree of intraspinal hemorrhaging (Supplemental Figure 2A). By 1 day after injury, blood neutrophil numbers were again reduced in both genotypes, likely reflecting a transient exhaustion of the primed reservoir and/or recruitment of mobilized BM neutrophils to the injured spinal parenchyma (see below); 
A
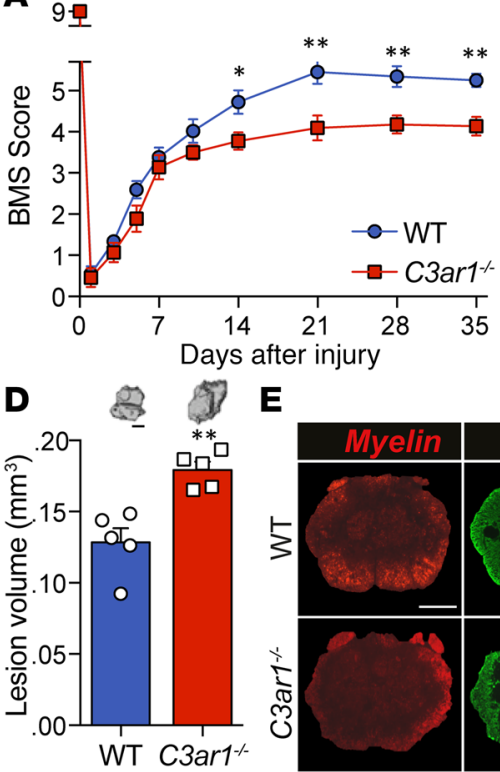

B

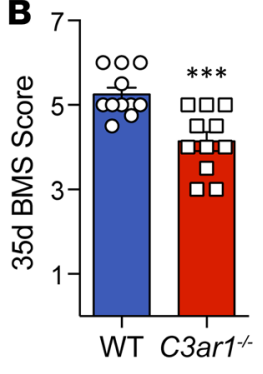

C
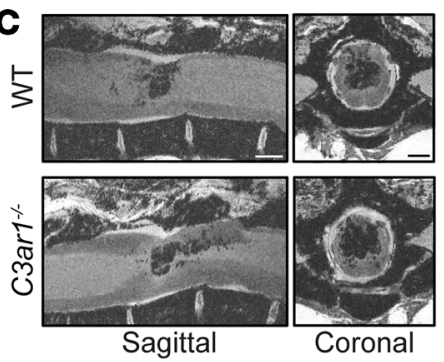

$\mathbf{F}$

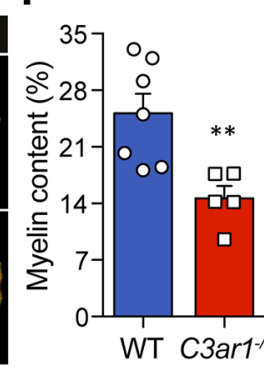

G

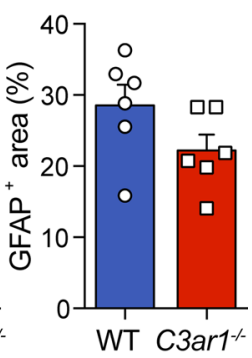

WT C3ar1 $\%$
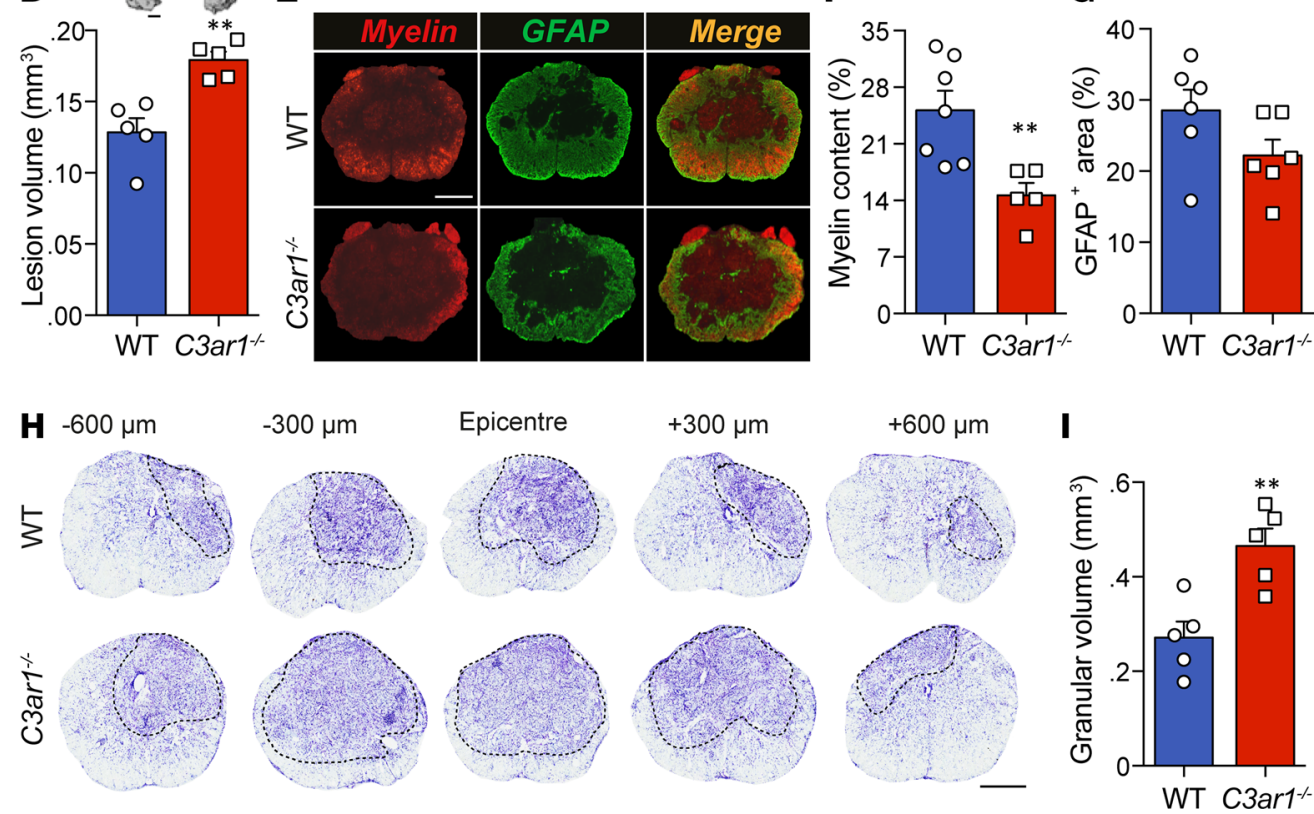

Figure 2. Neurological recovery from SCl is impaired in absence of C3aR1. (A) Open field locomotor (BMS) scores, showing the recovery of comparably injured WT and $\mathrm{C3arr}^{-1-}$ mice. Note that $\mathrm{C3arr}^{-1-}$ mice regained less hindlimb locomotor function and had significantly lower BMS scores at 14, 21, 28, and 35 days after SCI. Data points represent mean \pm SEM $(n=13-15) .{ }^{*} P<0.05 ;{ }^{* *} P<0.01$ by 2 -way repeated-measures ANOVA with Bonferroni's post hoc test (WT vs. C3arr ${ }^{-1-}$ mice at matching time points). (B) Bar graph showing endpoint BMS scores (mean \pm SEM), with data points representing individual mice within each cohort $(n=13-15)$. ${ }^{* *} P<0.001$ by 2 -tailed Student's $t$ test. (C) Representative images showing postmortem $\mathrm{T}_{2}{ }^{*}$-weighted spinal cord MRIs for WT and C3ar1 ${ }^{-/-}$mice at 35 days after injury. (D) Quantitative analysis of hypointense lesion core volumes (voxels) in WT and $C 3 a r^{1 /-}$ mice ( $n=5$ per group); representative $3 \mathrm{D}$ reconstructions of lesion cores are shown at the top of the graph for each genotype. ${ }^{* *} P<0.01$ by 2 -tailed Student's $t$ test. (E) Representative images of the lesion epicenter in WT and $\mathrm{C3arr}^{-1-}$ mice at 35 days after $\mathrm{SCl}$, showing myelin staining (red, left), GFAP immunoreactivity (green, middle), and a merged image (right). (F and $\mathbf{G}$ ) Quantitative analysis showing decreased myelin content $(\mathbf{F})$ but no difference in GFAP staining $(\mathbf{C})$ at the lesion epicenter in $\mathrm{CBarR}^{-1-}$ mice. ${ }^{* *} P<0.01$ by 2 -tailed Student's $t$ test. ( $\mathbf{H}$ and $\left.\mathbf{I}\right)$ Representative cresyl violet-stained sections and quantitative analysis showing an increased abnormal granular tissue presence in lesioned $C 3 a 1^{1--}$ spinal cord. ${ }^{* *} P<0.01$ by 2-tailed Student's $t$ test. Scale bars: $0.75 \mathrm{~mm}$ (sagittal) or $0.4 \mathrm{~mm}$ (coronal) (C), $50 \mu \mathrm{m}$ (D), $250 \mu \mathrm{m}$ (E), and $300 \mu \mathrm{m}$ (H).

these possibilities are not mutually exclusive. Otherwise, the influence of C3aR1 over early leukocyte mobilization (2 hours) appeared mostly specific to neutrophils, as no differences were observed for other WBC populations, including lymphocytes (WT $3.9 \pm 1.0 \times 10^{6} \mathrm{cells} / \mathrm{ml}$ vs. C3ar1 ${ }^{-/-} 4.2 \pm 0.8 \times 10^{6}$ cells $/ \mathrm{ml} ; P=0.88$ ) and $\mathrm{Gr}-1^{+} \mathrm{SSC}^{\text {lo }}$ inflammatory monocytes (WT $2.1 \pm 0.55 \times 10^{5} \mathrm{cells} / \mathrm{ml}$ vs. C3ar1 ${ }^{-1-}$ $\left.2.5 \pm 0.66 \times 10^{5} \mathrm{cells} / \mathrm{ml} ; P=0.67\right)$. Lastly, a splenic contribution to the acute SCI-induced neutrophil mobilization into the circulation can be ruled out, as granulocyte numbers for this organ in WT mice closely mirror blood data; that is, they are significantly increased over baseline ( $>7$-fold) at the 2-hour time point (SCI $1.41 \pm 0.18 \times 10^{6}$ vs. naive $0.18 \pm 0.04 \times 10^{6} \mathrm{CD}^{2} 1 \mathrm{~b}^{+} \mathrm{Ly}_{6} \mathrm{G}^{+} \mathrm{SSC}^{\mathrm{hi}}$ cells/spleen; $n=$ 5 per group, $P<0.0001)$. Collectively, these findings suggest that $\mathrm{C} 3 \mathrm{aR} 1$ negatively regulates the $\mathrm{BM}$ neutrophil mobilization response to (neuro-) trauma. 
A

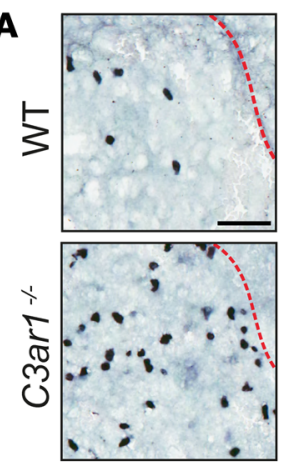

B
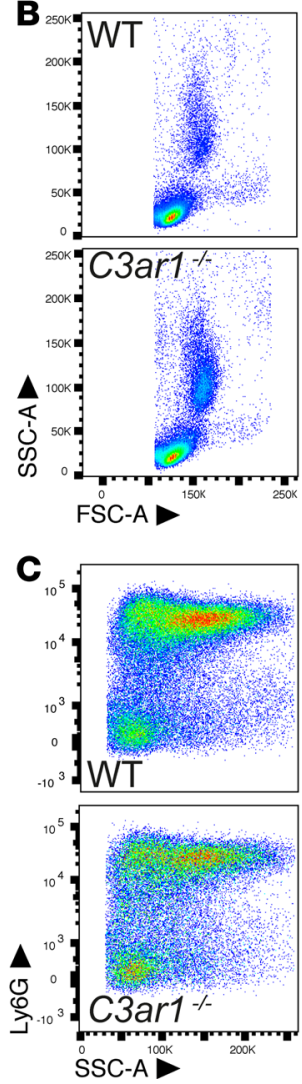

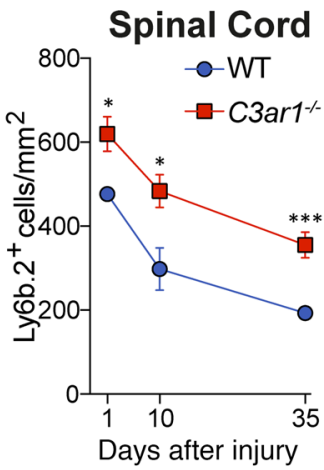

Blood

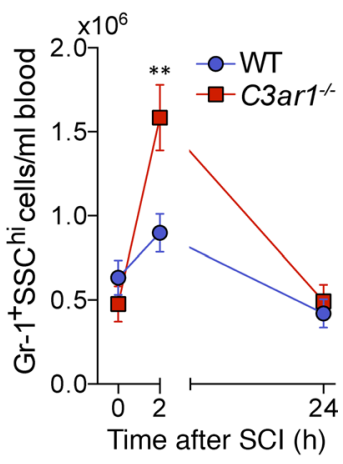

Bone Marrow

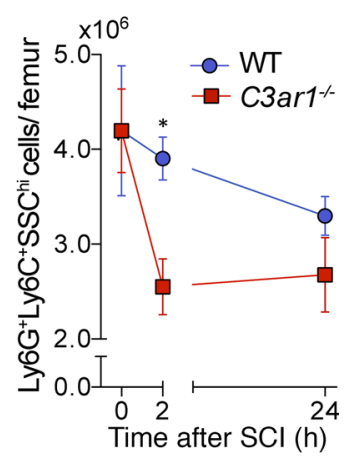

Figure 3. $\mathrm{Clar1}^{-/-}$mice have increased neutrophil recruitment and display acute granulocytosis in response to SCI. (A) Photomicrographs (left) and quantitative data (right) of the Ly6B.2+ infiltrate in WT and $\mathrm{CBar1}^{-/-}$mice. Dashed red lines indicate the boundary between the dorsal columns and horns ( 1 day after $\mathrm{SCI})$. Note that Ly6B. $2^{+}$cell numbers are significantly increased in the injured spinal cord of $\mathrm{Clarr}^{-/-}$mice at 1,10 , and 35 days after $\mathrm{SCl}$. Data points represent the mean \pm SEM $(n=5-8)$. Scale bar: $50 \mu \mathrm{m}$. (B) Quantitative analysis of circulating blood granulocyte numbers acutely after $\mathrm{SCl}$. Note the significant expansion of this population in $\mathrm{C3ar1}^{-1-}$ mice at 2 hours after $\mathrm{SCl}$. (C) BM granulocyte numbers were significantly lower in $\mathrm{Clar1}^{-1-}$ mice at 2 hours after $\mathrm{SCl}$ compared with their WT counterparts. Data points for $\mathbf{B}$ and $\mathbf{C}$ are mean \pm SEM $(n=$ 6 per genotype and time point). ${ }^{*} P<0.05 ;{ }^{*} P<0.01$; ${ }^{* * *} P<0.001$ by 2 -way ANOVA with Bonferroni's post hoc test for matching time points.

C3aR1 regulates BM cytokine production/availability. We next measured select complement proteins, key chemokines/cytokines, their receptors, and proteases to begin addressing how C3aR1 regulates BM neutrophil mobilization. Intraspinal and/or plasma protein levels of complement component $\mathrm{C} 5 \mathrm{a}$, its main signaling receptor C5aR1, CXCL1 (KC/IL-8), G-CSF, and CXCL12 (SDF-1) were all regulated by SCI but not differentially expressed between genotypes (Supplemental Figure 2, B-I). In the BM, however, CXCL1 was significantly increased in $C 3$ ar $^{-/-}$extracellular fluid at $2(P<0.05)$ and 24 hours after SCI $(P<0.0001$; Figure 4A). Surface expression of the main CXCL1 receptor, CXCR2, on BM neutrophils was not different between genotypes ( $P=0.47$; Supplemental Figure 3A). G-CSF, another key mobilizer, was similarly increased in BM extracellular fluid of $\mathrm{C}_{3} a{ }^{1^{-1}}$ mice, although only at 1 day after injury (1.5-fold, $P<0.01$; Figure 4B). The retention factor CXCL12 was also acutely increased in $\mathrm{C} \mathrm{ar} \mathrm{r}^{-1-} \mathrm{BM}$ extracellular fluid at 2 hours after SCI $(P<0.05$; Figure 4 C), but then fell (1.6-fold) to below WT levels by 1 day after SCI $(P<$ 0.05; Figure 4D). Like CXCR2, there was no difference in surface staining of CXCR4 (CXCL12 receptor) on BM neutrophils between WT and $C 3 a r 1^{-1-}$ mice $(P=0.48$; Supplemental Figure $3 \mathrm{~B})$. For complement, C3a levels in the BM extracellular fluid rapidly increased after SCI at $2(P<0.05)$ and 24 hours after injury $(P<0.001)$, but they were not different between genotypes (Figure $4 \mathrm{E})$. Levels of $\mathrm{C} 5 \mathrm{a}-\mathrm{a}$ known potent activator of neutrophils - were also not influenced here by C3aR1 deficiency (Supplemental Figure 3C). 
We lastly measured several key proteases involved in neutrophil egress from the BM. Of these, we found that only neutrophil elastase was increased in $\mathrm{C}_{3} \mathrm{arl}^{-1-} \mathrm{BM}$ extracellular following SCI (24 hours after injury; Supplemental Figure 3D). There was no effect of genotype on cathepsin G levels in BM extracellular fluid (Supplemental Figure 3E); MMP-9 activity, although generally lower in $C 3 \mathrm{arl}^{{ }^{-{ }_{-}}}$mice, was also similar between genotypes in terms of its responsiveness to SCI (Supplemental Figure 3, F and G; see complete unedited blots in the supplemental material). Collectively, these findings indicate that $\mathrm{C} 3 \mathrm{a}$ levels rapidly increase in BM extracellular fluid after SCI, and that $\mathrm{C} 3 \mathrm{aR} 1$ normally negatively regulates the expression of select neutrophil-mobilizing factors (i.e., CXCL1, G-CSF, and neutrophil elastase) following CNS injury.

CXCL1/CXCR2 signaling drives BM neutrophil mobilization in response to SCI. Given that CXCL1 was the only differentially expressed chemokine between WT and $C 3 a 1^{-/-} \mathrm{BM}$ at 2 hours after SCI, we sought to determine its role in neutrophil mobilization and the blood granulocytosis phenotype of $\mathrm{C}_{3} \mathrm{ar}^{-{ }^{--}}$mice. Administration of a selective antagonist for the main CXCL1 receptor (hereafter referred to as CXCR2-A) blocked BM neutrophil egress in response to SCI and, in doing so, also prevented the acute blood granulocytosis phenotype of $\mathrm{C}_{\mathrm{arr}} \mathrm{T}^{-/}$mice (Figure 5, A-C). These findings indicate that $\mathrm{C} 3 \mathrm{aR} 1$ acts as a negative regulator of CXCL1 expression and/or associated CXCR2 signaling, which in turn controls the magnitude of BM neutrophil mobilization following a major traumatic event like SCI.

Reinstatement of C3aR1 in the peripheral immune compartment, or neutrophil depletion, rescues the C3ar1 ${ }^{-1-}$ phenotype. We next hypothesized that if changes in BM neutrophil-mobilizing factors and the associated blood granulocytosis phenotype were directly influencing SCI outcomes, then reinstating the expression of C3aR1 within the peripheral immune compartment should counteract, at least in part, the impaired recovery of $\mathrm{C} \mathrm{arl}^{{ }^{-1}}$ animals. Transplantation of WT BM into conditioned $\mathrm{CBar}^{-1-}$ recipients indeed rescued their functional phenotype, with these mice now recovering to the same extent as WT counterparts (Figure 6, A and B). We also found that WT mice receiving a $C 3 a r 1^{-/-} \mathrm{BM}$ transplant phenocopied conventional $C 3 a r 1^{-/-}$mice, in that they displayed impaired recovery from SCI (Figure 6, A and B). These experiments demonstrate that presence of C3aR1 on circulating leukocytes, but not CNS-resident cells, is a critical determinant in the functional outcome from SCI.

To directly link increased neutrophil mobilization under C3aR1-deficient conditions to worsened outcomes from SCI, we next selectively depleted these cells in vivo via anti-Ly6G ( $\alpha$-Ly6G) antibody treatment. We hypothesized that if the magnitude of neutrophil mobilization acutely after SCI was indeed driving the impaired recovery of $\mathrm{C} \mathrm{ar} \mathrm{I}^{-1-}$ mice, then ablating these cells should restore BMS locomotor scores to WT levels. Flow cytometry confirmed the efficacy of $\alpha$-Ly6G-mediated neutrophil depletion at the level of the blood, BM, spleen, and injured spinal cord for up to at least 72 hours after SCI (Figure 7, A-F). Extending from earlier observations (Figure 3B), significant granulocytosis was again observed at 72 hours after SCI in C3ar1 ${ }^{-1-}$ mice treated with a nonspecific IgG2 $\alpha$ isotype (Iso) control antibody (C3ar1 ${ }^{-/-}$plus Iso, $9.4 \pm 0.79 \times$ $10^{5} \mathrm{cells} / \mathrm{ml}[n=3]$ vs. WT plus Iso, $\left.2.0 \pm 1.0 \times 10^{5} \mathrm{cells} / \mathrm{ml}[n=5] ; P<0.01\right)$. Regardless, $\alpha$-Ly6G antibody treatment depleted circulating SSChi Ly6C ${ }^{+}$cells with equal efficacy (WT plus $\alpha$-Ly6G, $0.025 \pm 0.014 \times 10^{5}$ cells/ml $[n=5]$ vs. $C 3$ arl $^{-1-}$ plus $\alpha$-Ly6G, $0.024 \pm 0.012 \times 10^{5}$ cells $\left./ \mathrm{ml}[n=3] ; P=0.27\right)$. Consistent with our earlier observations, $C 3 a r 1^{-1-}$ mice treated with isotype control antibody regained less hindlimb function than their WT counterparts $(P<0.05$; Figure 7, G and H). Treatment with $\alpha-L y 6 \mathrm{G}$, on the other hand, improved recovery of both WT and $C 3 a r 1^{-/-}$mice $(P<0.01)$, highlighting the injurious role of neutrophils in this condition. Most importantly, $\alpha$-Ly6G treatment enhanced the neurological recovery of $C 3 a r 1^{-/-}$mice to a level that was indistinguishable from their WT counterparts (Figure 7, G and H). Postmortem analysis of histopathology independently confirmed improved SCI outcomes based on myelin content within spared white matter, and also a full rescue of the $C 3 a^{-1-} 1^{-1}$ phenotype with $\alpha$-Ly6G treatment (Figure 7I). These findings thus provide direct evidence that the SCI-induced granulocytosis indeed drives the worsened neurological outcomes in $\mathrm{C}_{3} \mathrm{ar}^{-1-}$ mice, and also in more general terms that the extent of BM neutrophil mobilization has a direct negative impact on recovery from experimental SCI. This relationship was also found in human patients with traumatic SCI, as individuals with high circulating neutrophil numbers on admission were less likely to have a positive conversion in their neurological grading (American Spinal Injury Association Impairment Scale [AIS]) at discharge $(P<0.01$; Figure $7 \mathrm{~J})$. This difference remained when taking into account patient age, sex, admission AIS grade, and new injury severity scores (NISS) as possible confounders through logistic regression analysis (odds ratio $[\mathrm{OR}]=5.895 ; 95 \%$ confidence internal [CI] 1.421-24.453; $P<0.05)$. These findings thus support an injurious role for neutrophils in traumatic SCI that is conserved between mice and humans, and also highlight the importance of better understanding neutrophil mobilization (and function) for conditions that are hallmarked by systemic inflammation and granulocytosis. 
A

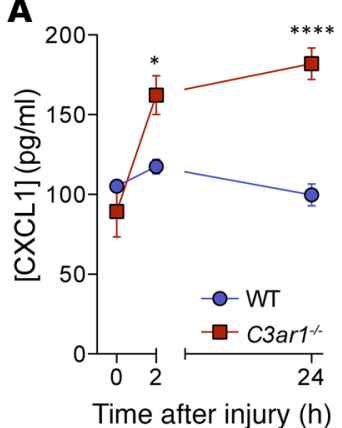

C

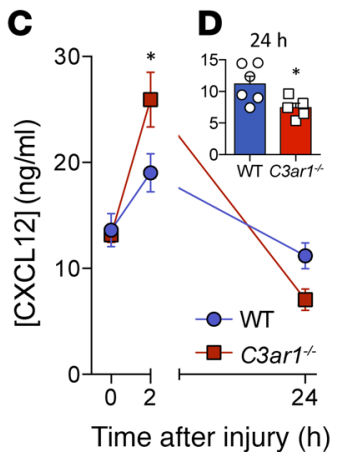

B

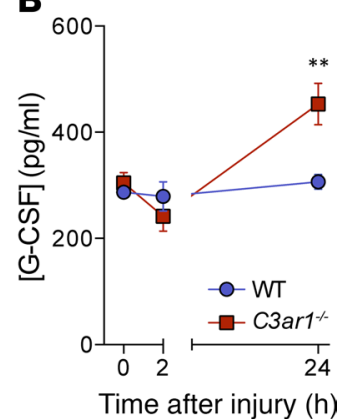

E

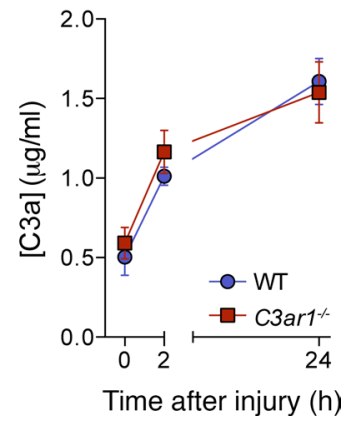

Figure 4. C3aR1 deficiency polarizes the BM extracellular environment to one that favors neutrophil mobilization. (A) CXCL1 levels were significantly elevated in $\mathrm{CBar1}^{-1-} \mathrm{BM}$ extracellular fluid at both 2 and 24 hours after SCI. (B) G-CSF levels in BM extracellular fluid were also significantly increased in C3ar7 $1 /$ mice, but only at the 24-hour time point. (C) Levels of the retention factor CXCL12 were acutely increased in response to $\mathrm{SCI}$ ( 2 hours), but below pre-injury and WT levels at 24 hours after injury (D). (E) Time course of C3a production in the BM niche of WT and $\mathrm{CZarr}^{-1-}$ mice following $\mathrm{SCI}$. For all graphs, data points (or bars) are mean \pm SEM ( $n=6$ per group). ${ }^{*} P<0.05$; ${ }^{* *} P<0.01 ;{ }^{* * *} P<0.0001$ by 2-way ANOVA with Bonferroni's post hoc test (A-C and $\mathbf{E}$ ) or 2-tailed Student's $t$ test (D).

We already explored here how early neutrophil depletion affects the recruitment of other immune cells during the acute, subacute, and intermediate phases of SCI. Of all the cell types examined, only the presence of a $\mathrm{B}$ cell subset $\left(\mathrm{CD} 45^{+} \mathrm{B} 220^{\text {mid }} \mathrm{CD} 11 \mathrm{c}^{-}\right.$cells) and monocytes/macrophages $\left(\mathrm{CD} 45^{\mathrm{hi}} \mathrm{CD} 11 \mathrm{~b}^{+} \mathrm{C}-\right.$ D64 ${ }^{\text {hi }}$ cells) was altered in $\alpha$-Ly6G-treated mice at 1 and 14 days after SCI, respectively $(P<0.05$; Supplemental Figure 4); no other differences were observed. These findings demonstrate that $\alpha$-Ly6G treatment early after SCI has only a very modest impact on the (subsequent) recruitment of other immune cells. The observed benefits of $\alpha$-Ly6G treatment therefore most likely result from an acute attenuation of secondary damage to the spinal cord that is caused directly by activated neutrophils themselves and/or the way in which they influence the activation states of other cells.

C3aR1 reduces cellular responsiveness to CXCL1/CXCR2 signaling. Having determined that eliminating $\mathrm{C} 3 \mathrm{aR} 1$ changes the BM microenvironment to one that favors neutrophil mobilization under challenged conditions (Figure 4), and also that the resulting granulocytosis phenotype could be alleviated with CXCR2 antagonism (Figure 5), we lastly investigated whether C3aR1 signaling may also directly regulate the responsiveness of neutrophils to mobilizing/chemotactic signals. In vitro chemotaxis assays revealed that the responsiveness of Ly6G-enriched mouse BM neutrophils toward CXCL1 was indeed suppressed $(P<$ 0.01) by exposure to the selective C3aR1 peptide agonist E7 (4) for WT but not C3ar1 ${ }^{-/-}$cells (Figure 8A). As E7 does not have any chemotactic effect on BM neutrophils (Supplemental Figure 5), these findings indicate that signaling events downstream of C3aR1 negatively regulate intracellular pathways activated by CXCL1. The same phenomenon was observed when complement activation product C5a was used as the chemoattractant (Supplemental Figure 5), indicating that these C3aR1 effects apply more broadly and are not just restricted to the CXCL1/CXCR2 axis.

Activation of the PI3K/AKT pathway downstream of GPCRs like CXCR2 (and C5aR1) plays a key role in controlling cell motility towards chemotactic signals (Figure 8B) (25), and stimulation of mouse BM neutrophils with CXCL1 indeed increased AKT phosphorylation as expected (Figure 8C). Importantly, however, this CXCL1-induced increase in phosphorylated AKT was fully annulled by E7 exposure (Figure $8, \mathrm{D}-\mathrm{F})$, and we further corroborated these findings by demonstrating similar responses in human neutrophils stimulated with IL-8 (Supplemental Figure 6).

With a recent study linking ERK phosphorylation to cell membrane recruitment of PTEN (26), a well-established negative regulator of the PI3K/AKT pathway, we wondered whether this phosphatase could be the missing link as to how C3aR1 is able to control the responsiveness of neutrophils to mobilizing/chemotactic signals. We first confirmed that activation of C3aR1 leads to ERK1/2 phosphorylation in 
A

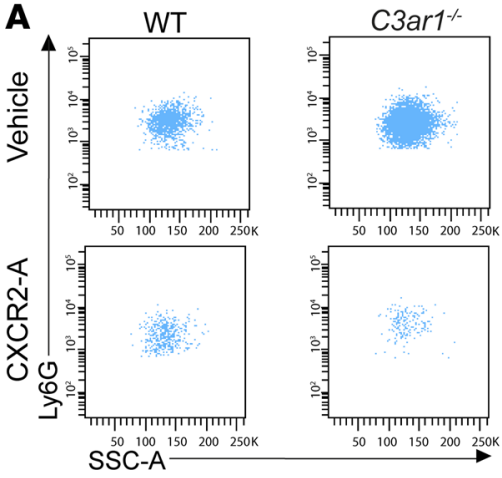

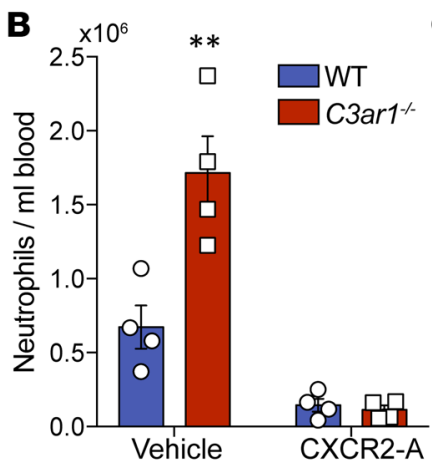

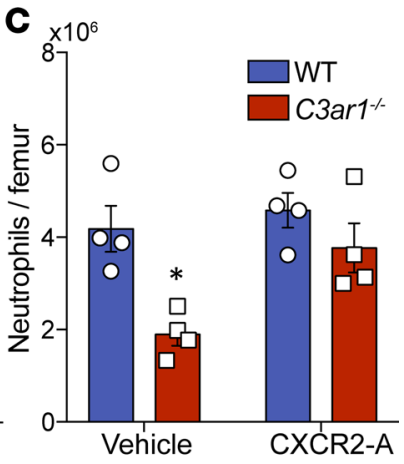

Figure 5. CXCR2 antagonism blocks SCI-induced BM neutrophil mobilization and granulocytosis in C3ar1 ${ }^{-/-}$mice. (A) Representative flow cytometry plots showing the Ly6 $\mathrm{C}^{+} S \mathrm{CC}^{\text {hi }}$ cell population in the blood of WT and $\mathrm{CZarr}^{-/-}$mice at 2 hours after $\mathrm{SCI}$, and following treatment with either vehicle (top) or CXCR2 antagonist (CXCR2-A; bottom). (B) Quantification of Ly6C $+5 S C C^{\text {hi }}$ cell numbers in the circulating blood. Note that CXCR2-A treatment rescues the SCl-induced granulocytosis phenotype of $\mathrm{CZar1}^{1 /-}$ mice. (C) CXCR2 antagonism retains neutrophils in the bone marrow of $\mathrm{C3ar7^{-1- }}$ mice with $\mathrm{SCl}$. Bar graphs are mean $\pm \mathrm{SEM}$, with data points representing individual mice within each cohort $(n=4$ per group). ${ }^{*} P<0.05 ;{ }^{* *} P<0.01$ by 2 -way ANOVA with Bonferroni's post hoc test.

neutrophils (Figure 8, G-I and Supplemental Figure 6A), and were also able to show that this change was associated with an increase in the geometric mean fluorescence intensity (MFI) for PTEN staining following E7 but not CXCL1 exposure (Figure 8, J-L).

We next employed in vitro chemotaxis assays again to directly probe a role for PTEN in mediating the negative regulatory effect of the $\mathrm{C} 3 \mathrm{a} / \mathrm{C} 3 \mathrm{aR} 1$ axis on mouse $\mathrm{BM}$ neutrophil migration. Addition of the PTEN inhibitor VO-OHpic (100 nM) (27) indeed completely reversed the C3aR1-mediated suppression of BM neutrophil chemotaxis, fully restoring the migration of these cells toward CXCL1; exposure to VO-OHpic alone (i.e., without E7-induced C3aR1 coactivation) did not change the migratory response (Figure 8M). Although we did not investigate other mouse strains of different genetic backgrounds (28), involvement of PTEN in the negative modulatory effect of the C3a/C3aR1 axis over chemotactic signals appears to be conserved between mice and humans. Specifically, the IL-8-induced increase in phosphorylated AKT was retained in the presence of E7 when the PTEN inhibitor VO-OHpic was also added (Supplemental Figure 6B). We finally theorized that if C3aR1 signaling controls BM neutrophil migration/ mobilization in a PTEN-dependent manner, then blocking the actions of this phosphatase in vivo should induce granulocytosis in WT mice that is akin to their $\mathrm{C}_{\text {arr }}{ }^{-/-}$counterparts. Administration of VO-OHpic ( $2 \mathrm{mg} / \mathrm{kg}$ i.v.) to WT SCI mice indeed led to greater neutrophil presence in the blood (Figure $8 \mathrm{~N}$ ), and their numbers were comparable to those seen in $\mathrm{C}_{3} \mathrm{ar}^{-1-}$ mice (see Figure 3B and Figure 5B). As VO-OHpic may also target SHP-1 (29), a separate cohort of mice was treated with the SHP-1/SHP-2 PTPase inhibitor NSC87877 (2.5 mg/kg i.p.). No change in neutrophil presence was seen at the level of the blood with this treatment (Supplemental Figure 7), strongly suggesting that the observed effects of VO-OHpic are indeed mediated via PTEN and not SHP-1.

\section{Discussion}

Excessive and/or deregulated complement system activation following trauma can increase host tissue damage, impair wound healing, and lead to unfavorable outcomes (30). Consistent with this, gene knockout or pharmacological approaches to inhibit complement at the level of C1q (31), factor B (32), C3 (33), $\mathrm{C} 3 \mathrm{~b} / \mathrm{C} 4 \mathrm{~b}$ (34), and C5a (35) all improve recovery from a major neurotraumatic event like SCI. However, no study to our knowledge has directly explored a putative role for C3a/C3aR1 in this condition. The mechanism via which $\mathrm{C} 3 \mathrm{aR} 1$ regulates $\mathrm{BM}$ neutrophil mobilization in response to tissue injury more generally has also remained elusive. Here, we identify the $\mathrm{C} 3 \mathrm{a} / \mathrm{C} 3 \mathrm{aR} 1$ axis as a negative regulator that controls expression of select neutrophil-mobilizing factors, particularly CXCL1, in the BM microenvironment. We further show that activation of the $\mathrm{C} 3 \mathrm{a} / \mathrm{C} 3 \mathrm{aR} 1$ axis controls the responsiveness of $\mathrm{BM}$ neutrophils to mobilizing factors like CXCL1. By acting as a physiological antagonist, C3a/C3aR1 limits neutrophil mobilization and associated inflammatory tissue pathology. 


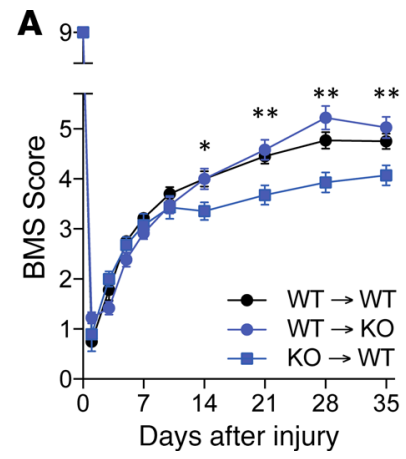

B

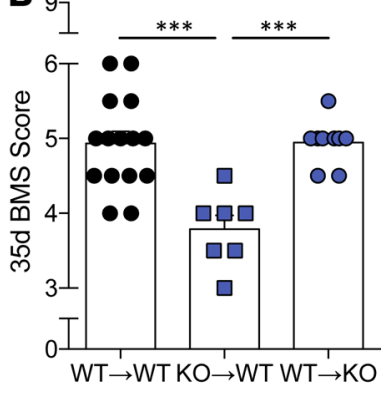

Figure 6. C3aR1 influences SCl outcomes via the peripheral immune compartment. (A) Functional recovery (BMS scores) of WT and $\mathrm{CBar1}^{-1-} \mathrm{BM}$ chimeric mice. Note that transplantation of WT bone marrow (BM) into $\mathrm{CZar1}^{-1-}$ recipient mice $[\mathrm{WT} \rightarrow \mathrm{KO}$ ] restores $\mathrm{SCl}$ recovery to that of [WT $\rightarrow \mathrm{WT}] \mathrm{BM}$ chimeras. WT mice receiving a $\mathrm{CZar1}^{-1-} \mathrm{BM}$ transplant (i.e., [KO $\left.\rightarrow \mathrm{WT}\right] \mathrm{BM}$ chimeras) phenocopied conventional $\mathrm{CZar1}^{-1-}$ mice, with a significantly worsened recovery from $\mathrm{SCl}$. Data points represent mean $\pm \mathrm{SEM}\left(n=7-15\right.$ per group). ${ }^{*} P<0.05 ;{ }^{*} P<$ 0.01 by 2 -way repeated measures ANOVA with Bonferroni's post hoc test comparing $[\mathrm{WT} \rightarrow \mathrm{KO}]$ and $[\mathrm{WT} \rightarrow \mathrm{WT}]$ with $[\mathrm{KO} \rightarrow \mathrm{WT}]$ mice. (B) Bar graph showing individual BMS scores for experimental animals within each group at the study endpoint ( 35 days after $\mathrm{SCI}$ ). Bar graphs are mean $\pm \mathrm{SEM}$, with data points representing individual mice within each cohort. ${ }^{* *} P<0.001$ by 1 -way ANOVA with Bonferroni's post hoc test.

Several other previous studies have positively implicated the C3a/C3aR1 axis in reparative and/or neuroprotective processes, including retinal regeneration (36), the expression of neurotropic factors by glia $(37,38)$, and neurogenesis (39). Although the present findings do not exclude a role for C3aR1 within the CNS, our BM chimera experiments showed that a loss of this receptor on CNS-resident cells does not change the neurological outcome from SCI. Rather, its expression within the peripheral immune compartment was identified as the crucial determinant for SCI recovery. Consistent with this, targeted depletion of neutrophils fully rescued the C3ar1 $1^{--}$phenotype and improved overall recovery. This outcome supports other studies that have negatively implicated neutrophils in secondary inflammatory pathology following experimental SCI (11-14), and we further corroborated this by linking the extent of SCI-induced neutrophil mobilization to recovery prognosis in human patients. For previous studies showing either no or even adverse effects of neutrophil depletion (40-42), it is worth noting that these mostly used the anti-Gr-1 antibody (RB6-8C5), which depletes not only neutrophils but also Ly6G-Ly6C $\mathrm{C}^{+}$monocytes/myeloid-derived suppressor cells (41), and can increase TNF responses (43).

Our animal experiments did not reveal any evidence for differential expression of neutrophil-mobilizing and/or -retention factors between genotypes under homeostatic conditions, or for altered maturation of BM neutrophils $(44,45)$ that could otherwise explain the acute granulocytosis seen in $\mathrm{C}^{-1 \mathrm{ar}^{-/-}}$mice after insult. Taken together with our previous observations that pharmacological activation of C3aR1 reduces neutrophil numbers in the blood in response to gut ischemia/reperfusion injury (4), the present findings therefore indicate a direct negative regulatory role for $\mathrm{C} 3 \mathrm{a} / \mathrm{C} 3 \mathrm{aR} 1$ in $\mathrm{BM}$ neutrophil mobilization under inflammatory conditions, as also supported by our in vitro chemotaxis experiments. To understand how $\mathrm{C} 3 \mathrm{a} / \mathrm{C} 3 \mathrm{aR} 1$ controls BM neutrophil egress, we screened a panel of proteins known to be associated with the retention, mobilization, and/or recruitment of these cells. These experiments revealed that the local $\mathrm{BM}$ microenvironment of injured $\mathrm{C}_{3} \mathrm{ar}^{-/-}$mice was changed toward one that is more favorable for neutrophil mobilization. In particular, we found that BM CXCL1 levels were abnormally elevated at 2 and 24 hours after SCI, and that blocking the main CXCL1 receptor, CXCR2, prevented the blood granulocytosis phenotype in $\mathrm{C}_{3} \mathrm{rr}^{-1-}$ mice. The observed key role for the CXCL1/CXCR2 axis in BM neutrophil mobilization after SCI is consistent with previous reports that CXCR2 deficiency overall causes neutropenia and retains neutrophils in the BM (46) and, further, that inhibition of this pathway alleviates inflammation in SCI (47) and other neutrophil-mediated pathologies (48). G-CSF levels were also increased in C3ar1 ${ }^{-1}$ mice, although not until 1 day after SCI. Consistent with the view that G-CSF can act cooperatively with CXCL1 to promote neutrophil mobilization by reducing CXCR4 signaling (44, 49-52), CXCL12 levels in the $\mathrm{BM}$ microenvironment of $\mathrm{C} \mathrm{arr}^{-/-}$mice were also lower at 1 day after SCI. Collectively, these findings suggest that $\mathrm{C} 3 \mathrm{a} / \mathrm{C} 3 \mathrm{aR} 1$ acts to constrain the levels of neutrophil-mobilizing factors within the BM niche in response to tissue injury and associated inflammation.

Finally, we also uncovered downstream signaling events via which C3aR1 directly modulates the sensitivity of neutrophils to chemotactic cues like CXCL1. Activation of PI3K $\gamma$ downstream of CXCR2 normally converts the membrane lipid phosphatidylinositol-3,4-bisphosphate $\left(\mathrm{PIP}_{2}\right)$ into phosphatidylinositol-3,4,5-triphosphate ( $\left.\mathrm{PIP}_{3}\right)$ at the leading edge of the neutrophil. Membrane-bound $\mathrm{PIP}_{3}$ then allows for the docking of Akt, triggering a series of signaling events that ultimately culminate in directional cell movement. Consistent with this, PI3K $\gamma$-deficient mice have limited recruitment of neutrophils and do not produce $\mathrm{PIP}_{3}$ or phosphorylated AKT following exposure to CXCL1, C5a, or fMLP in inflammatory pathologies such as septic peritonitis (53). Although we did not study the specific PI3K isoform involved, 

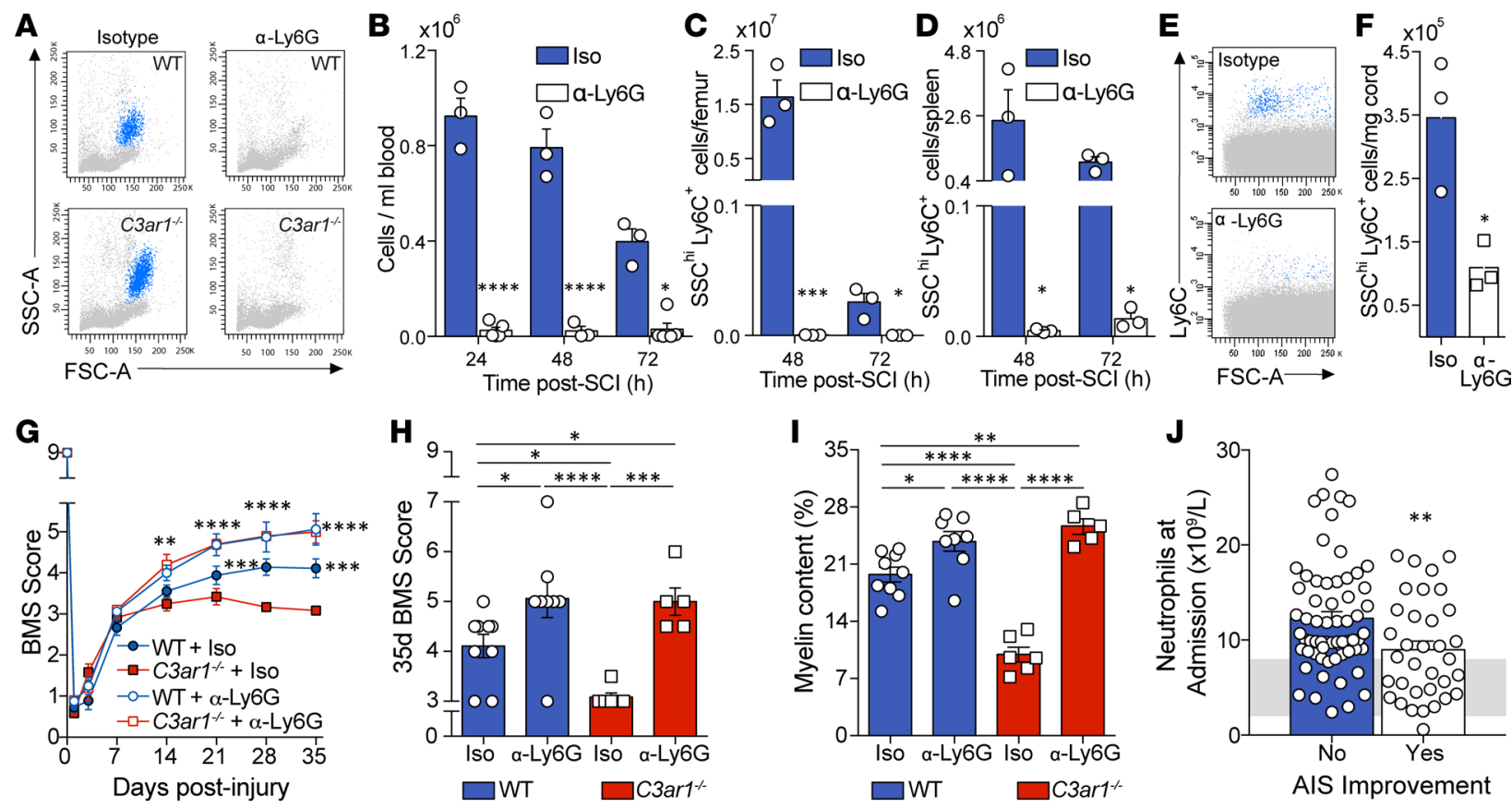

Figure 7. The extent of neutrophil mobilization is negatively correlated with outcomes in experimental and human SCI. (A) Representative flow cytometry plots showing that circulating Ly6C ${ }^{+} \mathrm{SSC}^{\text {hi }}$ cells (blue) remain present in mice treated with a nonspecific IgC2 $\alpha$ isotype control antibody (Iso, top row), but are effectively depleted for at least at 72 hours following anti-Ly6C antibody treatment ( $\alpha$-Ly6C, bottom row). (B) Quantitative data showing the impact of $\alpha$-Ly6C treatment on the number of circulating granulocytes (Ly6C $\mathrm{CSSC}^{\text {hi }}$ cells) up to 72 hours after SCI. (C-F) Ly6C ${ }^{+}$SSC ${ }^{\text {hi }}$ cells were also reduced in the BM (C), spleen (D), and spinal cord (E and $\mathbf{F}$ ) as a result of $\alpha$-LyGG treatment. Bar graphs are mean \pm SEM, with data points representing individual mice within each cohort ( $n=3-5$ per group). ${ }^{*} P<0.05 ;{ }^{* *} P<0.001$; ${ }^{* * *} P<0.0001$ by 2-way ANOVA with Bonferroni's post hoc test (B-D), or 2-tailed Student's $t$ test (F). (G) Depletion of Ly6 $\mathrm{C}^{+}$cells improves recovery from $\mathrm{SCl}$ and alleviates the functional phenotype of $\mathrm{CZar}^{-1-}$ mice. Data points represent mean \pm SEM ( $n=5-9$ per group). ${ }^{* *} P<0.01 ;{ }^{* *} P<0.001 ;{ }^{* * *} P<0.0001$ by repeated-measures 2-way ANOVA with Bonferroni's post hoc test (asterisks indicate significance vs. the "CZar1 ${ }^{-1-}+$ Iso" group). (H and I) Endpoint BMS scores $(\mathbf{H})$ and epicenter myelin content (I) at 35 days after SCI. Bar graphs are mean $\pm \mathrm{SEM}$, with data points representing individual mice within each cohort $(n=5-9)$. ${ }^{*} P<0.05 ;{ }^{* *} P<0.01 ;{ }^{* * *} P<0.001 ;{ }^{* * * *} P<0.0001$ by 1 -way ANOVA with Bonferroni's post hoc test. (J) Circulating neutrophil numbers at clinical presentation for SCI patients with and without a positive AIS grade conversion on discharge. ${ }^{* *} P<0.01$ by Mann-Whitney $U$ test.

our experiments show that C3aR1 signaling can negatively regulate CXCL1-induced AKT phosphorylation and BM neutrophil chemotaxis. We identified the phosphatase PTEN, a known negative regulator of the PI3K/AKT pathway that normally localizes to the sides and back of the crawling cell where it converts $\mathrm{PIP}_{3}$ back to $\mathrm{PIP}_{2}$ in areas away from the leading edge (54), as the likely intermediary via which C3aR1 limits BM neutrophil chemotaxis/egress in vitro and in vivo. Overall, our studies uncover what we believe is a new negative regulatory mechanism that connects rapid injury-induced complement activation and associated C3aR1 signaling in the BM microenvironment with the magnitude of the ensuing cellular inflammatory response (Supplemental Figure 8).

The discovery of how C3aR1 controls acute inflammation and neutrophil responsiveness following injury has clear translational significance. Specifically, it identifies agonism of C3aR1, or its downstream intermediary PTEN, as a therapeutic option to broadly inhibit neutrophil mobilization/recruitment in response to multiple chemotactic signals (e.g., CXCL1 and C5a) for a range of inflammatory conditions where these cells have been associated with pathology (2). Our findings in relation to PTEN further warn against broad/global inhibition of this phosphatase as a means to promote CNS axon regeneration (55), as this would also increase the magnitude of the peripheral inflammatory response and thus likely be detrimental for recovery. Indeed, previous studies have shown that loss of PTEN activity enhances neutrophil recruitment to sites of inflammation in neutropenia-associated pneumonia (56), and similar observations were made here in the context of SCI. In summary, our findings highlight the importance of understanding the role of complement activation products like $\mathrm{C} 3 \mathrm{a}$ across multiple tissue compartments. They also warrant further research into the therapeutic development and use of C3aR1 agonists to attenuate neutrophil-driven pathology in complex trauma cases like SCI and other inflammatory conditions. 
A

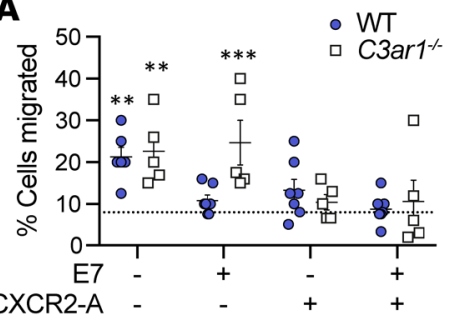

D

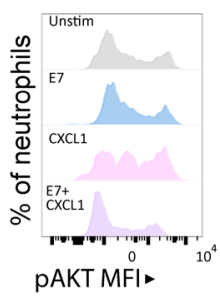

G

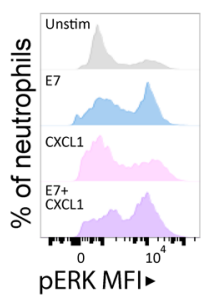

J

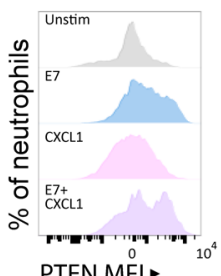
PTEN MFI .

M

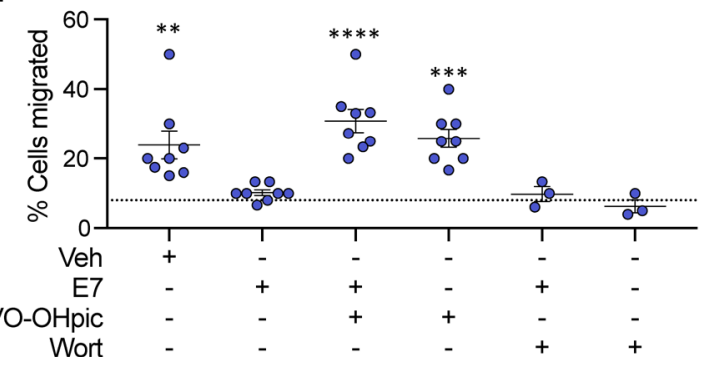

B

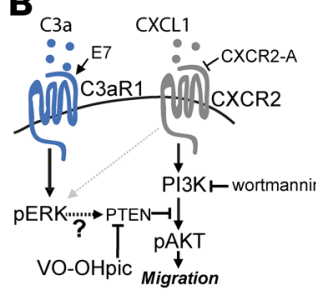

C
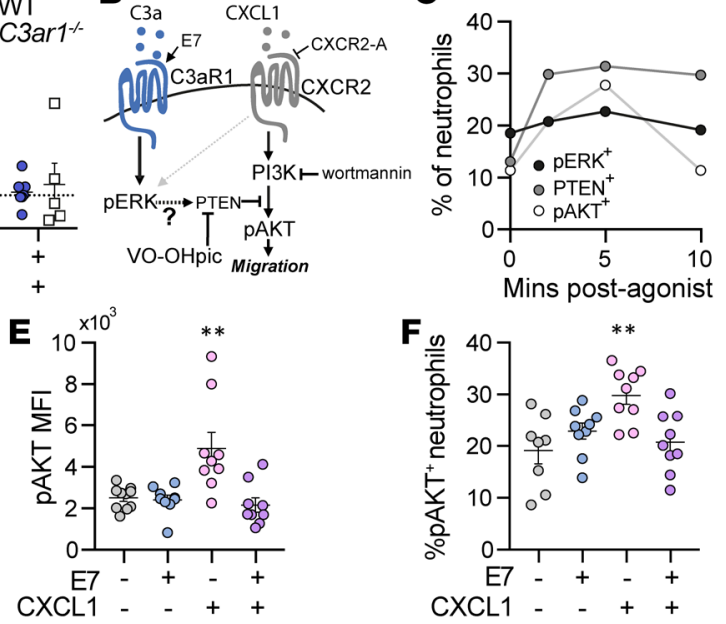

H

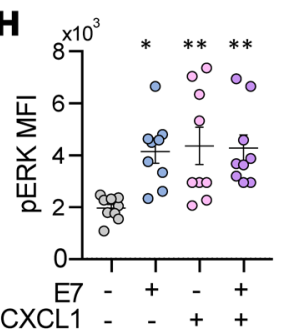

$\mathbf{K}$

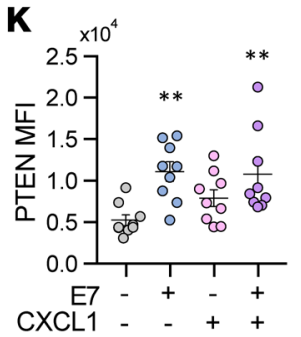

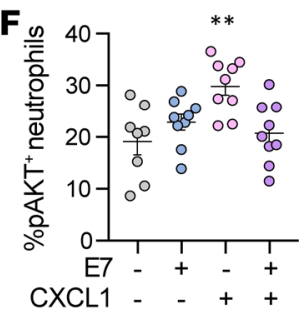

I

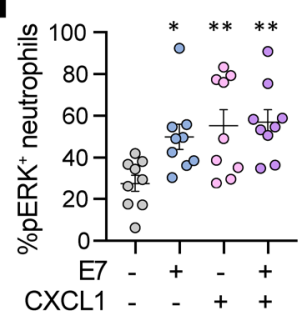

$\mathbf{L}$

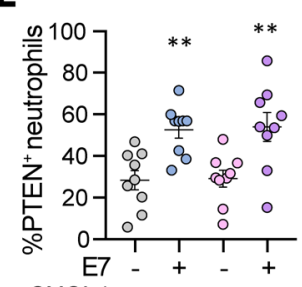

CXCL1 - - + +

$\mathbf{N}$

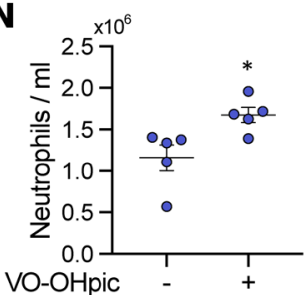

Figure 8. C3aR1 controls the responsiveness of BM neutrophils to chemotactic signals. (A) Chemotaxis assays of Ly6C-enriched BM neutrophils toward CXCL1. Note that CXCL1-induced cell migration is selectively abolished in WT mice with C3aR1 coactivation (E7 treatment) and/or CXCR2 antagonism (CXCR2-A). Error bars are SEM ( $n=5-7$ per group). ${ }^{* *} P<0.01 ;{ }^{* *} P<0.001$ by 2-way ANOVA with Dunnett's post hoc test against baseline (i.e., no CXCL1; dashed line). (B) Working model as to how $[$ 3aR1 might regulate $C X C L 1 / C X C R 2$-mediated $\mathrm{BM}$ neutrophil chemotaxis, and the site of action for the various inhibitors used. (C) Time course showing the percentage of BM neutrophils staining positive for phosphorylated ERK (p-ERK) and PTEN following E7 exposure, or phosphorylated AKT ( $p$-AKT) after CXCL1 stimulation. (D-F) Overlays showing the geometric mean fluorescence intensity (MFI) for p-AKT staining in BM neutrophils at baseline (unstimulated) and under test conditions (D). Exposure to CXCL1, but not E7, increases both the MFI (E) and overall percentage (F) of p-AKT ${ }^{+}$ BM neutrophils. C3aR1 agonism with E7 prevents these CXCL1-induced changes in AKT phosphorylation/ positivity. (G-I) Overlays showing the MFI for p-ERK staining in BM neutrophils at baseline (unstimulated) and under test conditions (G). Activation of C3aR1 and CXCR2 with E7 and CXCL1, respectively, (or a combination thereof) increases both the MFI (H) and overall percentage (I) of $\mathrm{p}-\mathrm{ERK}^{+} \mathrm{BM}$ neutrophils. (J-L) Overlays showing PTEN MFI in BM neutrophils at baseline (unstimulated) and under test conditions (J). Activation of C3aR1 with E7 increases both the MFI (K) and overall percentage of PTEN $^{+}$BM neutrophils (L). Error bars for $\mathbf{E}, \mathbf{F}, \mathbf{H}, \mathbf{I}, \mathbf{K}$, and $\mathbf{L}$ are SEM ( $n=9$ per group). ${ }^{*} P<0.05$; ${ }^{* *} P<0.01$ by 1 -way ANOVA with Dunnett's post hoc test compared with unstimulated controls. (M) PTEN inhibition with $\mathrm{VO}-\mathrm{OHpic}$ (100 nM) overcomes the E7/C3aR1-mediated inhibition of BM neutrophil responsiveness to CXCL1. Treatment with wortmannin $(100 \mathrm{nM})$ confirmed a key role of the PI3K/ AKT pathway in mediating the chemotactic response of BM neutrophils to CXCL1. Error bars are SEM ( $n=3-8$ per group). ${ }^{* *} P<0.01$; ${ }^{* *} P<0.001 ;{ }^{* * *} P<0.0001$ by 1 -way ANOVA with Dunnett's post hoc test versus baseline (i.e., no CXCL1; dashed line). (N) Administration of VO-OHpic to WT SCI mice leads to increased mobilization/circulating neutrophil numbers (2 hours after surgery; data are from 3 independent experiments). Error bars are SEM ( $n=5$ per group). ${ }^{*} P<0.05$ by 2 -tailed Student's $t$ test.

\section{Methods}

Mice

Adult female C57BL/6J (WT, $n=271), C_{3 a r 1^{---}}(n=123)(57), C_{5 a r 1^{--}}(n=14)(58)$, and Cx3cr ${ }^{85 p /+}$ mice $(n$ = 3) (59) were used; genetically modified lines had been previously backcrossed for more than 10 generations onto a C57BL/6J genetic background. Mice were obtained from local breeding colonies at The University of Queensland's Biological Resources Facility, or the Animal Resources Centre (Perth, Western Australia), and housed in clean holding facilities on a 12-hour light/dark cycle with unlimited access to food and water. Mice were matched by age and weight within experiments. All experiments were conducted in accordance with the Australian Code for the Care and Use of Animals for Scientific Purposes, as well as the ARRIVE guidelines (60) with regards to randomization (order of surgery, treatment allocation) and blinding (surgery and analysis). 
$\mathrm{SCl}$

Mice were anesthetized with tiletamine/zolazepam (50 mg/kg; Virbac) and xylazine (10 mg/kg; Troy Laboratories) and subjected to a 70-kilodyne contusive SCI using the infinite horizon impactor (61). Postoperatively, animals received a single injection of buprenorphine $(0.5 \mathrm{mg} / \mathrm{kg} \mathrm{s.c.})$ in Hartmann's solution (Baxter) for analgesia, and were administered $1.0 \mathrm{mg} / \mathrm{kg}$ gentamicin s.c. for the first 5 days after injury. Bladders were manually voided twice daily for the duration of the experiments.

\section{C3a ELISA}

WT mice were subjected to either SCI or sham (i.e., laminectomy) surgery and then killed with sodium pentobarbitone (100 mg/kg i.p., Virbac) at 30 minutes, 2 hours, 6 hours, 12 hours, 1 day, 4 days, 7 days, or 35 days after injury ( $n=4-5$ per time point). Naive mice were used to assess baseline C3a (time point 0 ). Blood and spinal cord samples were collected and processed as described previously (35). C3a concentrations in blood plasma and spinal cord samples were measured as per the manufacturer's instructions (BD Biosciences; 558250, 558251, and 558618). For spinal cord, C3a levels were divided by the total protein content of the sample (Thermo Fisher Scientific, Pierce BCA protein assay, 23227).

\section{C3aR1 expression}

Mice were sacrificed using sodium pentobarbitone $(100 \mathrm{mg} / \mathrm{kg}$ i.p., Virbac) and transcardially perfused with $20 \mathrm{ml}$ of saline $(0.9 \% \mathrm{NaCl})$ containing $10 \mathrm{IU} / \mathrm{ml}$ heparin (Pfizer) and $2 \% \mathrm{NaNO}_{3}$, followed by $30 \mathrm{ml}$ of phosphate-buffered Zamboni's fixative (2\% picric acid, $2 \%$ formaldehyde, $\mathrm{pH} 7.2-7.4$ ). The vertebral column was then dissected out and postfixed overnight at $4^{\circ} \mathrm{C}$. Spines were imaged where applicable (see below, MRI), dissected from the vertebral column, and cryoprotected via subsequent overnight incubations in $10 \%$ and $30 \%$ sucrose solutions. Spinal cords were then embedded in OCT compound (ProSciTech) and snap-frozen. Coronal sections $(20 \mu \mathrm{m})$ were cut using a Leica cryostat CM3050-S and collected (1:5 series) on Superfrost Plus slides (Lomb Scientific).

Immunofluorescent staining was used to visualize C3aR1 in the injured spinal cord (chicken antiC3aR1, 1:200; BMA Biomedicals, T-2301), along with neutrophils (rat anti-Ly6B.2, 1:400; AbD Serotec, clone 7/4), myeloid cells (rat anti-CD11b, 1:200; AbD Serotec, clone 5C6), microglia/macrophages (rabbit anti-Iba1, 1:500; Wako Pure Chemical Industries, 019-19741), and/or astrocytes (mouse anti-glial fibrillary acidic protein [GFAP], 1:1000; BD Biosciences, 556330). Tissue from injured $C x 3 \mathrm{cr} 1^{g f p /+}$ mice was used to confirm that Ly6B.2 staining was confined to neutrophils at 1 day after SCI. Staining procedures were as described previously (35), using goat anti-chicken IgY-Alexa Fluor 555 (1:500; Invitrogen, A32932), donkey anti-rat IgG-Alexa Fluor 488 (1:200; Jackson ImmunoResearch, 712-546-153), goat anti-rabbit IgG-Alexa Fluor 647 (1:250; Invitrogen, A-21245), or goat anti-mouse IgG-Alexa Fluor 488 (1:200; Invitrogen, A-11001) secondary antibodies. C3aR1 antibody specificity was confirmed on injured C3ar1 ${ }^{-1-}$ spinal cord tissue. Images were captured on an Olympus BX61 confocal microscope and analyzed using Imaris (v. 7.7.0; Bitplane).

\section{Assessment of functional recovery}

Open field locomotor performance was assessed using the 10-point BMS (23). For the initial WT versus

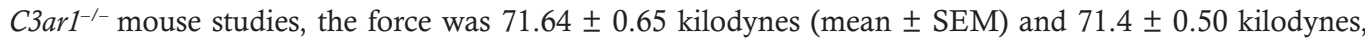
with a tissue displacement of $586.5 \pm 7.6 \mu \mathrm{m}$ and $588.8 \pm 19.1 \mu \mathrm{m}$, respectively. Injury parameters were not different between groups $(P>0.05)$.

\section{MRI}

Vertebral columns with the spinal cord in situ were washed in 0.1 M PBS, followed by immersion for 48 hours in PBS containing gadolinium contrast agent (0.2\% Magnevist, Bayer HealthCare Pharmaceuticals). Samples were imaged on a 16.4-T small animal MRI (Bruker BioSpin) and lesion volumes analyzed using AVIZO 6.2 software (Visualisation Sciences Group) as detailed previously (35).

\section{Histopathology}

Nissl staining. Cresyl violet stain was performed to examine gross morphology of injured spinal cords. Areas densely populated by small $\mathrm{Niss}^{+}$bodies were outlined in ImageJ (NIH). The volume of granular tissue was estimated by multiplying the granular area by the section thickness. 
Myelin preservation and glial scarring. FluoroMyelin Red (1:150; Molecular Probes, 34652) was combined with rabbit anti-GFAP (1:1000; Dako, Z033401-2) and a goat anti-rabbit IgG-Alexa Fluor 488 secondary antibody (1:250; Invitrogen, A-11008). Stained spinal cord sections were photographed using an Olympus SZX12 Research Fluorescence Stereo Microscope (Spectra Services), and the proportional area of myelin and GFAP staining measured as described previously (35). The lesion epicenter was defined as the section with the least amount of FluoroMyelin Red staining relative to the section area.

Cellular inflammation. Immunohistochemical staining was performed as detailed previously (20) using rat anti-Ly6B.2, rabbit anti-Iba1, and rat anti-CD3 (1:400; BD Biosciences, clone 17A2). Slides were digitized using a ScanScope XT scanner (Aperio) with ImageScope software (Leica Biosystems). Ly6B.2 $2^{+}$ and $\mathrm{CD}^{+}$cell numbers were counted using the manual tag function in ImagePro Plus Version 6.3 (Media Cybernetics) and expressed as the number of cells per $\mathrm{mm}^{2}$ based on the section area. The Iba1 $1^{+}$infiltrate was quantified as the proportional area of stained tissue (8).

\section{Flow cytometry}

For quantification of circulating neutrophil numbers, $50 \mu 1$ of blood was obtained via retro-orbital eye bleeds from WT $(n=6)$ and $C 3 a r 1^{-1-}$ mice $(n=6)$ at 1 week prior to SCI, then again at 2 hours after SCI and 1 day after SCI. For each time point, equal numbers of mice from each genotype were always processed side by side. Mice were euthanized with sodium pentobarbitone immediately after their final eye bleed at 1 day after SCI. Blood was always collected and processed at the same time of day to minimize the impact of circadian variations (62). Samples were diluted 1:3 in anticoagulant buffer (DPBS with 4 mM EDTA, $\mathrm{pH} 7.2)$ on ice, then diluted 1:5 with red blood cell lysis buffer $\left(0.85 \% \mathrm{NH}_{4} \mathrm{Cl}, \mathrm{pH} 7.2\right)$ and incubated for 5 minutes at room temperature. Cells were centrifuged ( $300 \mathrm{~g}$ for 10 minutes) and resuspended in PBS with Zombie Green viability dye (BioLegend) for dead cell exclusion. Cells were resuspended to a concentration of $1 \times 10^{7}$ cells $/ \mathrm{ml}$ in cytometry blocking buffer $(0.5 \%$ BSA, 2 mM EDTA, in DPBS, $\mathrm{pH}$ 7.2), then incubated with rat anti-CD16/32 (1:200; BD Biosciences, clone 2.4G2) for 10 minutes on ice. Biotinylated rat anti-Gr-1 (1:400; BD Biosciences, clone RB6-8C5) was added for 10 minutes, followed by streptavidin-Alexa Fluor 546 (1:400; Invitrogen, S11225). Cells were centrifuged (300 $\mathrm{g}$ for 10 minutes), washed, and resuspended in $395 \mu 1$ DPBS with 2 mM EDTA. Flow-Count Fluorospheres ( $5 \mu 1 /$ sample, Beckman Coulter) were added as an internal standard to quantify absolute cell numbers. Samples were analyzed using an LSRII flow cytometer (BD Biosciences) and BD FACS Diva/ FlowJo software. Compensation was applied to remove spectral overlap. Doublets were excluded based on FSC-A/FSC-H linearity. Unstained and isotype controls were used to confirm staining specificity, and to determine gate placement. Similar procedures were used to determine C5aR1 expression using an Alexa Fluor 647-conjugated rat anti-mouse C5aR1 (1:20; BD Biosciences, clone 10/92). Isotype control antibodies (BD Biosciences), unstained samples, as well as blood from $C 5 \mathrm{ar} 1^{-/-}$mice, were used to confirm antibody specificity.

For quantification of BM neutrophil numbers, WT and $C 3 a r 1^{-1-}$ mice were sacrificed at 2 and 24 hours after SCI with sodium pentobarbitone. Naive mice from each genotype were used for assessing BM cellularity under steady-state conditions. BM was flushed from femurs with ice-cold anticoagulant buffer and then passed through a $40-\mu \mathrm{m}$ cell strainer. Cells were centrifuged at $500 \mathrm{~g}$ for 5 minutes and resuspended in $5 \mathrm{ml}$ of $\mathrm{RBC}$ lysis buffer. Further processing was similar to that described for blood samples, with the additional inclusion of Zombie Violet (BioLegend), Ly6G-Alexa Fluor 647 (1:200; BD Biosciences, clone 1A8), CXCR2-PE (1:100; R\&D Systems, clone 242216), and CXCR4-PerCP-eFluor710 (1:200; eBioscience, clone 2B11).

\section{Spinal cord and plasma protein analysis}

C5a and cytokine levels. WT and C3ar1 ${ }^{-1-}$ mice were killed with sodium pentobarbitone at 2 hours after surgery. ELISAs were used to determine the levels of C5a (R\&D Systems, DY2150), CXCL1/KC (R\&D Systems, DY453), and G-CSF (R\&D Systems, MCS00) in each sample. For spinal cord samples, measured $\mathrm{C} 5 \mathrm{a}$ and cytokine concentrations were divided by the total protein content of the sample.

Hemoglobin. Spinal cord samples were diluted 1:30 in Drabkin's reagent (Sigma-Aldrich) and analyzed alongside a standard curve of bovine hemoglobin (Sigma-Aldrich). Samples were run in duplicate and absorbance measured at $540 \mathrm{~nm}$ using a Tecan Sunrise 96-well plate reader. Hemoglobin test results were divided by the total protein content of the sample. 


\section{BM protein analysis}

Complement and cytokines. BM extracellular fluids were collected from naive and SCI mice ( 2 and 24 hours after injury). Both femurs were flushed with $1 \mathrm{ml}$ of complement/protease inhibitor solution (1 mM PMSF in DMSO, $1 \% \mathrm{v} / \mathrm{v}$ protease inhibitor cocktail [Sigma-Aldrich], $100 \mu \mathrm{g} / \mathrm{ml}$ FUT175 [BD Biosciences] in 1× PBS, pH 7.4). Samples were centrifuged twice at $500 \mathrm{~g}$ for 10 minutes, and the supernatants were collected and stored at $-80^{\circ} \mathrm{C}$. C3a, C5a, G-CSF, CXCL1/KC, and CXCL12 (R\&D Systems, DY460) levels were measured via ELISA.

Proteases. Neutrophil elastase, cathepsin G, and MMP-9 were also quantified in BM extracellular fluids of WT and $C 3 a r 1^{-1-}$ mice ( $n=6$ per genotype and time point/condition). In brief, femurs were flushed with ice-cold PBS. Neutrophil elastase and cathepsin G concentrations were assessed spectrophotometrically using respective enzyme substrates, i.e., $0.8 \mathrm{mM}$ elastase substrate 1 (Merck) or $0.8 \mathrm{mM}$ cathepsin $\mathrm{G}$ substrate 1 (Calbiochem) alongside serial standards $(0.0375-1 \mu \mathrm{g} / \mathrm{ml}$ neutrophil elastase; $0.015-2 \mu \mathrm{g} / \mathrm{ml}$ cathepsin G; Elastin Products Co.). Samples were run in duplicate and absorbance measured after 3 hours at $405 \mathrm{~nm}$ on a FLUOstar plate reader. Protease concentrations in each sample were calculated after correcting for the background color of BM fluid. For MMP-9 analysis, $10 \mu 1$ of sample from each group was pooled and the average concentration between genotypes and conditions assessed via gel zymography (63).

\section{In vivo CXCR2 antagonism}

WT and $\mathrm{CBar1}^{-/-}$mice were treated with SB225002 (Tocris; $4 \mathrm{mg} / \mathrm{kg}$ i.p.) 1 hour before SCI. At 2 hours after injury, blood and BM samples were collected and processed for flow cytometry as described above.

\section{BM chimeras}

BM chimeras $[W T \rightarrow W T](n=15),\left[W T \rightarrow C 3 a r 1^{-1-}\right](n=9)$, and $\left[C 3 a r 1^{-1-} \rightarrow W T\right](n=7)$ were generated as detailed previously (35). Mice recovered for 8 weeks before undergoing SCI and BMS scoring. The average applied force was $72.58 \pm 0.56$ kilodynes (mean $\pm \mathrm{SEM}$ ) for [WT $\rightarrow W T], 71.57 \pm 0.65$ kilodynes for [C3ar1 ${ }^{--} \rightarrow W T$ ] , and $71.86 \pm 0.83$ kilodynes for [WT $\rightarrow{\mathrm{C} 3 a r 1^{-1}}^{-}$; the associated tissue displacement was $570.2 \pm 19.5 \mu \mathrm{m}, 573.4 \pm 17.9 \mu \mathrm{m}$, and $579.1 \pm 15.7 \mu \mathrm{m}$, respectively. Of note, 2 separate cohorts of $[W T \rightarrow W T]$ BM chimeras were generated as the appropriate control group for $[K O \rightarrow W T]$ and $[W T \rightarrow K O]$ BM chimeras, respectively. BMS scores of the $2[W T \rightarrow W T]$ BM chimera cohorts were pooled because their recovery was the same, and the statistical outcome was not affected by this.

\section{Neutrophil depletion}

WT and $C 3 a r 1^{--}$mice were injected with a rat $\alpha$-Ly6G antibody (BioXcell, clone 1A8; $2.5 \mathrm{mg} / \mathrm{kg}$ i.p.), or IgG2 $\alpha$ isotype (Iso) control (BioXcell, clone $2 \mathrm{~A} 3 ; 2.5 \mathrm{mg} / \mathrm{kg}$ i.p.) 3 hours before injury. Antibodies were custom made to contain less than 0.5 endotoxin units $(\mathrm{EU}) / \mathrm{mg}$. Surgical procedures, assessment of neurological recovery, and tissue pathology were all as detailed above. For functional experiments, the average applied force was $71.86 \pm 0.14$ kilodynes for WT plus Iso, $71.25 \pm 0.45$ kilodynes for WT plus $\alpha$-Ly6G, $71.50 \pm 0.62$ kilodynes for $\mathrm{C} \mathrm{arr}^{-/-}$plus Iso, and $71.83 \pm 0.54$ kilodynes for $C 3 \mathrm{ar} 1^{-/-}$plus $\alpha$-Ly6G; the associated tissue displacement was $541.3 \pm 14.28 \mu \mathrm{m}, 546.6 \pm 20.13 \mu \mathrm{m}, 552.3 \pm 38.48 \mu \mathrm{m}$, and $541.8 \pm 20.79 \mu \mathrm{m}$, respectively The success of neutrophil depletion with $\alpha$-Ly6G treatment was confirmed at the level of the blood, BM, spleen and spinal cord via flow cytometry as per established protocols (34). Neutrophils were gated as the Ly6C ${ }^{+} S_{S C} C^{\text {hi }}$ population (Ly6C-Alexa Fluor V450; BD Biosciences, clone AL-21). Additional multicolor flow cytometry was performed to characterize how neutrophil depletion affected the overall inflammatory infiltrate during the acute ( 1 day), subacute ( 7 days), and intermediate ( 14 days) phases of SCI ( $n=6$ per time point and condition; see Supplemental Figure 4). Delineated WBC populations were normalized to microglia numbers to account for variations in the yield/recovery of viable cells from different samples and/or experimental repeats (64). The following antibodies were used as appropriate for cell identification: CD45 PerCP-Cy5.5 or APC-Cy7 (BD Biosciences, clone 30-F11); CD64 PE-Cy7 (BioLegend, clone X54-5/7.1); Ly6C V450 (BD Horizon, clone AL-21) or Ly6C BV605 (BioLegend, clone HK1.4); CD115 PE (BioLegend, clone AFS98); CD11c PerCP-Cy5.5 (BioLegend, clone N418); MHC-II FITC (BioLegend, clone M5/114-15.12); NK1.1 APC (BioLegend, clone PK136); CD3e BV711 (BD Biosciences, clone 145-2C11); CD4 FITC (BD Biosciences, clone RM4-5); CD8 BV500 (BD Biosciences, clone 53-6.7); CD69 BV421 (BioLegend, clone H1.2F3); B220 BV786 (BD Horizon, clone RA3-6B2); and Zombie Red (BioLegend, 423110) for live/dead staining. 


\section{Mouse chemotaxis assays}

BM neutrophils were isolated from the femurs and tibiae of WT and $C 3 a r 1^{-1-}$ mice using the $\alpha$-Ly6G microbead kit (Miltenyi Biotec, 130-092-332) and MiniMACS cell separation system. Migration assays were performed in duplicate using a modified Boyden chamber (Neuroprobe), which consisted of a 3 - $\mu$ m pore filter above a 96-well chamber. RPMI medium (31 $\mu 1)$ with or without recombinant mouse CXCL1 (R\&D Systems, 453-KC; $30 \mathrm{nM}$ ) was added to wells below the filter. The filter was snapped in place and $5 \times 10^{4}$ neutrophils in $20 \mu 1$ of RPMI were added to the hydrophobic rings above the filter, allowing contact with the media below. For C3aR1 activation, Ly6G-enriched cells from WT, $\mathrm{C}_{\mathrm{ar}} \mathrm{I}^{-1-}$,

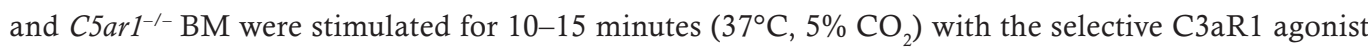
WWGKKYRASKLGLAR $(\mathrm{E} 7,10 \mu \mathrm{M})$ prior to being added to the migration plate. Similar procedures were followed for inhibition of CXCR2 (Tocris Bioscience, SB225002; $10 \mu \mathrm{M}$ ), PTEN (Sigma-Aldrich, V8639; $100 \mathrm{nM}$ ), or pan-PI3K (Sigma-Aldrich, W3144; $100 \mathrm{nM}$ ). Plates were incubated for 1 hour $\left(37^{\circ} \mathrm{C}, 5 \% \mathrm{CO}_{2}\right)$, after which the filter was carefully removed and the number of transmigrated cells in the bottom compartment counted following dilution in trypan blue solution (1:1) using a Neubauer hemocytometer. This method was also used to evaluate in vitro chemotaxis of BM neutrophils toward E7 or C5a (BioVision Inc, 4895A; $50 \mathrm{nM}$ ).

\section{Intracellular flow cytometry}

Mice were anesthetized with sodium pentobarbitone. BM from femurs and tibiae was flushed with $2 \mathrm{mM}$ EDTA in DPBS (2.68 mM KCl, $1.47 \mathrm{mM} \mathrm{KH}_{2} \mathrm{PO}_{4}, 137.89 \mathrm{mM} \mathrm{NaCl}, 8.10 \mathrm{mM} \mathrm{Na}_{2} \mathrm{HPO}_{4}$ ). Cells were washed by gentle centrifugation $\left(300 \mathrm{~g}, 5\right.$ minutes, $\left.4^{\circ} \mathrm{C}\right)$ and resuspended at a concentration of $2 \times 10^{6}$ cells $/ \mathrm{ml}$. Appropriate tubes were incubated with Zombie NIR (BioLegend) for 20 minutes at $4^{\circ} \mathrm{C}$. Cells were washed, centrifuged $\left(300 \mathrm{~g}, 5\right.$ minutes, $\left.4^{\circ} \mathrm{C}\right)$, and resuspended in IMDM containing $0.2 \% \mathrm{BSA}$ and anti-CD16/32 for 10 minutes on ice. Next, cells were incubated with antibodies against Ly6G-BUV395 (1:100), Ly6C-V450, (1:100), F4/80-PE (1:30; Bio-Rad, clone CI:A3-1), and CD11b-PE-CY7 (1:200; BD Biosciences, clone M1/70) for 20 minutes on ice. Cells were washed in IMDM plus $0.2 \%$ BSA, and then prewarmed $\left(10\right.$ minutes, $37^{\circ} \mathrm{C}, 5 \% \mathrm{CO}_{2}$ ) before stimulation with CXCL1, E7, combinations thereof, or vehicle (DMSO/saline). Next, ice-cold PBS with HALT protease/phosphatase inhibitor cocktail (1:100, Thermo Fisher Scientific) was added, after which the cells were washed and fixed (4\% paraformaldehyde in DPBS) for 20 minutes on ice. Cells were then resuspended in 90\% methanol with anti-CD16/32 (1:200) for 10 minutes on ice, followed by incubation with antibodies against phospho-p44/42 MAPK (ERK1/2) (Thr202/Tyr204)-Alexa Fluor 488 (Cell Signaling Technology, 4344; 1:50) and PTEN-Alexa Fluor 647 (BD Biosciences, 560003; 1:20), or phospho-AKT-Alexa Fluor 647 (Cell Signaling Technology, 4075; 1:50) for 20 minutes on ice. Cells were washed before resuspension in $2 \mathrm{mM}$ EDTA-containing DPBS and flow cytometry analysis.

\section{In vivo PTEN and SHP-1 antagonism}

WT mice were administered VO-OHpic (Sigma-Aldrich, V8639; 2 mg/kg i.v.), NSC87877 (Tocris; 2.5 mg/ $\mathrm{kg}$ i.p.), or vehicle (DMSO in saline or PBS) at 12 hours and 30 minutes prior to SCI ( $n=5$ per group). At 2 hours after SCI, $100 \mu \mathrm{l}$ of blood was collected via retro-orbital eye bleed and circulating neutrophil numbers quantified by flow cytometry as detailed above.

\section{Human SCI data}

Retrospective data were collected from a total of 97 SCI patients admitted to the Princess Alexandra Hospital in Brisbane between 2012 and 2017. For each patient, the extent of neurological impairment was assessed using the AIS grading criteria during the first week of admission to hospital (baseline) and then again at discharge. All patients had an AIS grade of A, B, or C upon admission; the neurological level of injury ranged between the cervical (62.8\%), thoracic (34.0\%), and lumbar $(2.1 \%)$ region of the spinal cord. Circulating neutrophil numbers were obtained from WBC differentials in admission blood samples (Pathology Queensland). There were no significant differences between patients who showed either no improvement $(n=63)$ or an AIS grade conversion $(n=34)$ with regard to their age (37.5 years vs. 37.9 years, respectively; $P=0.9009)$ or sex $(84.1 \%$ vs. $88.2 \%$ male, respectively; $P=0.7645)$. 


\section{Human neutrophil phospho-AKT assays}

Peripheral blood neutrophils were isolated from $25 \mathrm{ml}$ of whole blood that was collected by venipuncture from 4 healthy human volunteers ( 3 females and 1 male; age: $24.5 \pm 1.2$ years [mean \pm SD]); EDTA (1.8 $\mathrm{mg} / \mathrm{ml}$ ) was used as the anticoagulant. Blood was layered over a double gradient of Histopaque-1077 and Histopaque-1119 (Sigma-Aldrich) for density centrifugation ( $700 \mathrm{~g}, 30$ minutes at room temperature). The blood plasma and peripheral blood mononuclear cell layer were carefully removed, and the polymorphonuclear cell layer collected from the interface between Histopaque-1077 and Histopaque-1119; residual erythrocytes were removed through hypotonic lysis (65). Isolated neutrophils were resuspended in $2 \mathrm{ml}$ of Hank's balanced salt solution (Gibco), supplemented with calcium, magnesium, 25 mM HEPES, and 0.5 $\%$ BSA, then counted using a TC20 automatic cell counter (Bio-Rad). Cell viability and purity were $95.2 \%$ $\pm 0.5 \%$ and $97.4 \% \pm 0.6 \%$ (mean \pm SEM), respectively.

For phospho-AKT analysis, neutrophils were plated into a 96-well plate (500,000 cells per well), after which loaded plates were prewarmed for 25 minutes $\left(37^{\circ} \mathrm{C}, 5 \% \mathrm{CO}_{2}\right)$. Isolated neutrophils were incubated for 30 minutes with the C3aR1 agonist E7 $(10 \mu \mathrm{M})$ and/or PTEN inhibitor (VO-OHpic, $100 \mathrm{nM}$ ), then stimulated with $1 \mu \mathrm{g} / \mathrm{ml}$ IL-8 for 5-10 minutes and snap-frozen. Cells were homogenized by adding AlphaLISA SureFire Ultra 5× lysis/activation buffer mix (10:1 ratio; PerkinElmer) containing 1\% Protease Inhibitor Cocktail Set III (Calbiochem) at $4^{\circ} \mathrm{C}$ for 30 minutes. Cell lysates were frozen and stored at $-35^{\circ} \mathrm{C}$ until further use. Phospho-AKT levels for each sample were measured using the AlphaLISA Surefire Ultra p-AKT1/2/3 (Ser473) Assay kit (PerkinElmer).

\section{Statistics}

GraphPad Prism was used for data visualization and statistical analyses. BMS data sets were analyzed using repeated-measures 2-way ANOVA and Bonferroni's post hoc test. Differences in lesion volumes, histological data, flow cytometry, protein concentrations, and cell migration between experimental conditions and/or genotype were determined using a 2-sided Student's $t$ test, Mann-Whitney $U$ test, or 1-way or 2-way ANOVA with post hoc test as specified in the figure legends. All data sets are presented as mean and SEM, with statistical significance at $P<0.05$. Appropriate sample sizes were determined/confirmed via both a priori and post hoc power analysis, with the level of power $(1-\beta)$ set at 0.80 and $\alpha=0.05$. Logistic regression analysis was employed to assess the incidence of AIS conversion in human SCI patients, using circulating neutrophil numbers in admission blood samples as the predictor variable. The model was adjusted for patient age, sex, admission AIS grade, and NISS (SAS software version 9.4, SAS Institute Inc.).

\section{Study approval}

All experimental animal work was approved by The University of Queensland's Animal Ethics Committee (Anatomical Biosciences). Human studies involving retrospective analysis of SCI patient data and the collection of blood from healthy donors were approved by the Queensland Government's Human Research Ethics Committee for Metro South Health (HREC/16/QPAH/196) and The University of Queensland (HREC 2014000031). All individuals involved consented to having their data and/or blood samples used for research purposes.

\section{Author contributions}

FHB, SMT, JPL, TMW, and MJR conceived the study. FHB, TJ, ERG, LVB, XXL, BN, EJ, and MJR performed experiments and/or collected clinical data. FHB, TJ, ERG, XXL, JPL, TMW, and MJR analyzed data. GMW, GWO, FAM, KEC, KPAM, and JPL assisted with experimental design and/or data interpretation. FHB and MJR wrote the manuscript. All authors contributed to editing of the manuscript and approved the final version.

\section{Acknowledgments}

Work in the laboratory of MJR is supported by SpinalCure Australia, the Wings for Life Spinal Cord Research Foundation, and National Health and Medical Research Council of Australia (NHMRC; project grants 1060538 and 1163835). JPL was supported by an NHMRC Research Fellowship (grant 1044091), and TMW by an NHMRC Career Development Fellowship (grant 1105420), and Project Grant (number 1082271). FHB was supported by an Australian Postgraduate Award (University of Queensland). The authors further wish to express their gratitude to Virginia Nink (Queensland Brain Institute, University of 
Queensland) for assistance with flow cytometry, Luke Hammond (Queensland Brain Institute, University of Queensland) for expert assistance with microscopy and image acquisition, Kate Schroder (Institute for Molecular Bioscience, University of Queensland) for advice on neutrophil depletion experiments, Elissa Scriven (Princess Alexandra Hospital, Brisbane) for assistance with accessing trauma registry data, and University of Queensland Biological Resources staff for mouse colony maintenance and animal husbandry.

Address correspondence to: Marc J. Ruitenberg, School of Biomedical Sciences, The University of Queensland, Brisbane, QLD 4072, Australia. Phone: 61.7.3346.7602; Email: m.ruitenberg@uq.edu.au.

FHB's present address is: Department of Neuroscience, Center for Brain and Spinal Cord Repair, Wexner Medical Center, The Ohio State University, Columbus, Ohio, USA.

1. Furze RC, Rankin SM. Neutrophil mobilization and clearance in the bone marrow. Immunology. 2008;125(3):281-288

2. Soehnlein O, Steffens S, Hidalgo A, Weber C. Neutrophils as protagonists and targets in chronic inflammation. Nat Rev Immunol. 2017;17(4):248-261.

3. Kolaczkowska E, Kubes P. Neutrophil recruitment and function in health and inflammation. Nat Rev Immunol. 2013;13(3):159-175.

4. Wu MC, et al. The receptor for complement component C3a mediates protection from intestinal ischemia-reperfusion injuries by inhibiting neutrophil mobilization. Proc Natl Acad Sci USA. 2013;110(23):9439-9444.

5. Coulthard LG, Woodruff TM. Is the complement activation product C3a a proinflammatory molecule? Re-evaluating the evidence and the myth. J Immunol. 2015;194(8):3542-3548.

6. Wysoczynski M, Reca R, Lee H, Wu W, Ratajczak J, Ratajczak MZ. Defective engraftment of C3aR ${ }^{-/}$hematopoietic stem progenitor cells shows a novel role of the C3a-C3aR axis in bone marrow homing. Leukemia. 2009;23(8):1455-1461.

7. Ratajczak J, et al. Mobilization studies in mice deficient in either C3 or C3a receptor (C3aR) reveal a novel role for complement in retention of hematopoietic stem/progenitor cells in bone marrow. Blood. 2004;103(6):2071-2078.

8. Kigerl KA, McGaughy VM, Popovich PG. Comparative analysis of lesion development and intraspinal inflammation in four strains of mice following spinal contusion injury. J Comp Neurol. 2006;494(4):578-594.

9. Beck KD, Nguyen HX, Galvan MD, Salazar DL, Woodruff TM, Anderson AJ. Quantitative analysis of cellular inflammation after traumatic spinal cord injury: evidence for a multiphasic inflammatory response in the acute to chronic environment. Brain. 2010;133(Pt 2):433-447.

10. Fleming JC, et al. The cellular inflammatory response in human spinal cords after injury. Brain. 2006;129(Pt 12):3249-3269

11. Fleming JC, Bao F, Chen Y, Hamilton EF, Relton JK, Weaver LC. Alpha4beta1 integrin blockade after spinal cord injury decreases damage and improves neurological function. Exp Neurol. 2008;214(2):147-159.

12. Taoka Y, et al. Role of neutrophils in spinal cord injury in the rat. Neuroscience. 1997;79(4):1177-1182.

13. Saiwai H, et al. The LTB4-BLT1 axis mediates neutrophil infiltration and secondary injury in experimental spinal cord injury. Am J Pathol. 2010;176(5):2352-2366.

14. Gris D, et al. Transient blockade of the CD11d/CD18 integrin reduces secondary damage after spinal cord injury, improving sensory, autonomic, and motor function. J Neurosci. 2004;24(16):4043-4051.

15. Ahuja CS, et al. Traumatic spinal cord injury. Nat Rev Dis Primers. 2017;3:17018.

16. Quell KM, et al. Monitoring C3aR expression using a floxed tdTomato-C3aR reporter knock-in mouse. J Immunol. 2017;199(2):688-706

17. Martin U, et al. The human C3a receptor is expressed on neutrophils and monocytes, but not on B or T lymphocytes. J Exp Med. 1997;186(2):199-207.

18. Ischenko A, et al. Expression of a functional anaphylatoxin C3a receptor by astrocytes. J Neurochem. 1998;71(6):2487-2496.

19. Davoust N, Jones J, Stahel PF, Ames RS, Barnum SR. Receptor for the C3a anaphylatoxin is expressed by neurons and glial cells. Glia. 1999;26(3):201-211.

20. Carlson SL, Parrish ME, Springer JE, Doty K, Dossett L. Acute inflammatory response in spinal cord following impact injury. Exp Neurol. 1998;151(1):77-88.

21. Guglietta S, et al. Coagulation induced by C3aR-dependent NETosis drives protumorigenic neutrophils during small intestinal tumorigenesis. Nat Commun. 2016;7:11037.

22. Brennan FH, Cowin GJ, Kurniawan ND, Ruitenberg MJ. Longitudinal assessment of white matter pathology in the injured mouse spinal cord through ultra-high field (16.4 T) in vivo diffusion tensor imaging. Neuroimage. 2013;82:574-585.

23. Basso DM, Fisher LC, Anderson AJ, Jakeman LB, McTigue DM, Popovich PG. Basso Mouse Scale for locomotion detects differences in recovery after spinal cord injury in five common mouse strains. J Neurotrauma. 2006;23(5):635-659.

24. Bresnahan JC, Beattie MS, Todd FD, Noyes DH. A behavioral and anatomical analysis of spinal cord injury produced by a feedback-controlled impaction device. Exp Neurol. 1987;95(3):548-570.

25. Kölsch V, Charest PG, Firtel RA. The regulation of cell motility and chemotaxis by phospholipid signaling. J Cell Sci. 2008;121(Pt 5):551-559.

26. Zmajkovicova K, Jesenberger V, Catalanotti F, Baumgartner C, Reyes G, Baccarini M. MEK1 is required for PTEN membrane recruitment, AKT regulation, and the maintenance of peripheral tolerance. Mol Cell. 2013;50(1):43-55.

27. Mak LH, Vilar R, Woscholski R. Characterisation of the PTEN inhibitor VO-OHpic. J Chem Biol. 2010;3(4):157-163.

28. Wende $\mathrm{E}$, et al. The complement anaphylatoxin $\mathrm{C} 3 \mathrm{a}$ receptor $(\mathrm{C} 3 \mathrm{aR})$ contributes to the inflammatory response in dextran sulfate sodium (DSS)-induced colitis in mice. PLoS ONE. 2013;8(4):e62257.

29. Spinelli L, Lindsay YE, Leslie NR. PTEN inhibitors: an evaluation of current compounds. Adv Biol Regul. 2015;57:102-111. 
30. Brennan FH, Lee JD, Ruitenberg MJ, Woodruff TM. Therapeutic targeting of complement to modify disease course and improve outcomes in neurological conditions. Semin Immunol. 2016;28(3):292-308.

31. Galvan MD, et al. Deficiency in complement C1q improves histological and functional locomotor outcome after spinal cord injury. J Neurosci. 2008;28(51):13876-13888.

32. Qiao F, et al. The alternative and terminal pathways of complement mediate post-traumatic spinal cord inflammation and injury. Am J Pathol. 2010;177(6):3061-3070.

33. Ankeny DP, Guan Z, Popovich PG. B cells produce pathogenic antibodies and impair recovery after spinal cord injury in mice. J Clin Invest. 2009;119(10):2990-2999.

34. Qiao F, Atkinson C, Song H, Pannu R, Singh I, Tomlinson S. Complement plays an important role in spinal cord injury and represents a therapeutic target for improving recovery following trauma. Am J Pathol. 2006;169(3):1039-1047.

35. Brennan $\mathrm{FH}$, et al. The complement receptor C5aR controls acute inflammation and astrogliosis following spinal cord injury. J Neurosci. 2015;35(16):6517-6531.

36. Haynes T, et al. Complement anaphylatoxin C3a is a potent inducer of embryonic chick retina regeneration. Nat Commun $2013 ; 4: 2312$.

37. Jauneau AC, et al. Interleukin-1beta and anaphylatoxins exert a synergistic effect on NGF expression by astrocytes. $J$ Neuroinflammation. 2006;3:8.

38. Heese K, Hock C, Otten U. Inflammatory signals induce neurotrophin expression in human microglial cells. J Neurochem. 1998;70(2):699-707.

39. Rahpeymai Y, et al. Complement: a novel factor in basal and ischemia-induced neurogenesis. EMBO J. 2006;25(6):1364-1374

40. Lee SM, Rosen S, Weinstein P, van Rooijen N, Noble-Haeusslein LJ. Prevention of both neutrophil and monocyte recruitment promotes recovery after spinal cord injury. J Neurotrauma. 2011;28(9):1893-1907.

41. Saiwai H, et al. Ly6 $\mathrm{C}^{+}$Ly6G myeloid-derived suppressor cells play a critical role in the resolution of acute inflammation and the subsequent tissue repair process after spinal cord injury. J Neurochem. 2013;125(1):74-88.

42. Stirling DP, Liu S, Kubes P, Yong VW. Depletion of Ly6G/Gr-1 leukocytes after spinal cord injury in mice alters wound healing and worsens neurological outcome. J Neurosci. 2009;29(3):753-764.

43. Daley JM, Thomay AA, Connolly MD, Reichner JS, Albina JE. Use of Ly6G-specific monoclonal antibody to deplete neutrophils in mice. J Leukoc Biol. 2008;83(1):64-70.

44. Martin C, Burdon PC, Bridger G, Gutierrez-Ramos JC, Williams TJ, Rankin SM. Chemokines acting via CXCR2 and CXCR4 control the release of neutrophils from the bone marrow and their return following senescence. Immunity. 2003;19(4):583-593.

45. Suratt BT, et al. Role of the CXCR4/SDF-1 chemokine axis in circulating neutrophil homeostasis. Blood. 2004;104(2):565-571.

46. Eash KJ, Greenbaum AM, Gopalan PK, Link DC. CXCR2 and CXCR4 antagonistically regulate neutrophil trafficking from murine bone marrow. J Clin Invest. 2010;120(7):2423-2431.

47. Gorio A, et al. Reparixin, an inhibitor of CXCR2 function, attenuates inflammatory responses and promotes recovery of function after traumatic lesion to the spinal cord. J Pharmacol Exp Ther. 2007;322(3):973-981.

48. Mukaida N, Matsumoto T, Yokoi K, Harada A, Matsushima K. Inhibition of neutrophil-mediated acute inflammation injury by an antibody against interleukin-8 (IL-8). Inflamm Res. 1998;47 Suppl 3:S151-S157.

49. Lévesque JP, Hendy J, Takamatsu Y, Simmons PJ, Bendall LJ. Disruption of the CXCR4/CXCL12 chemotactic interaction during hematopoietic stem cell mobilization induced by GCSF or cyclophosphamide. J Clin Invest. 2003;111(2):187-196.

50. Petit I, et al. G-CSF induces stem cell mobilization by decreasing bone marrow SDF-1 and up-regulating CXCR4. Nat Immunol. 2002;3(7):687-694.

51. Semerad CL, Liu F, Gregory AD, Stumpf K, Link DC. G-CSF is an essential regulator of neutrophil trafficking from the bone marrow to the blood. Immunity. 2002;17(4):413-423.

52. Wengner AM, Pitchford SC, Furze RC, Rankin SM. The coordinated action of G-CSF and ELR + CXC chemokines in neutrophil mobilization during acute inflammation. Blood. 2008;111(1):42-49.

53. Hirsch E, et al. Central role for $\mathrm{G}$ protein-coupled phosphoinositide 3-kinase gamma in inflammation. Science. 2000;287(5455):1049-1053.

54. Phillipson M, Kubes P. The neutrophil in vascular inflammation. Nat Med. 2011;17(11):1381-1390.

55. Liu K, et al. PTEN deletion enhances the regenerative ability of adult corticospinal neurons. Nat Neurosci. 2010;13(9):1075-1081.

56. Li Y, et al. Targeted deletion of tumor suppressor PTEN augments neutrophil function and enhances host defense in neutropenia-associated pneumonia. Blood. 2009;113(20):4930-4941.

57. Kildsgaard J, Hollmann TJ, Matthews KW, Bian K, Murad F, Wetsel RA. Cutting edge: targeted disruption of the C3a receptor gene demonstrates a novel protective anti-inflammatory role for C3a in endotoxin-shock. J Immunol. 2000;165(10):5406-5409.

58. Hollmann TJ, Mueller-Ortiz SL, Braun MC, Wetsel RA. Disruption of the C5a receptor gene increases resistance to acute Gram-negative bacteremia and endotoxic shock: opposing roles of C3a and C5a. Mol Immunol. 2008;45(7):1907-1915

59. Jung S, et al. Analysis of fractalkine receptor CX(3)CR1 function by targeted deletion and green fluorescent protein reporter gene insertion. Mol Cell Biol. 2000;20(11):4106-4114.

60. Kilkenny C, Browne WJ, Cuthill IC, Emerson M, Altman DG. Improving bioscience research reporting: the ARRIVE guidelines for reporting animal research. PLoS Biol. 2010;8(6):e1000412.

61. Scheff SW, Rabchevsky AG, Fugaccia I, Main JA, Lumpp JE. Experimental modeling of spinal cord injury: characterization of a force-defined injury device. J Neurotrauma. 2003;20(2):179-193.

62. Scheiermann C, et al. Adrenergic nerves govern circadian leukocyte recruitment to tissues. Immunity. 2012;37(2):290-301.

63. Brenner CA, Adler RR, Rappolee DA, Pedersen RA, Werb Z. Genes for extracellular-matrix-degrading metalloproteinases and their inhibitor, TIMP, are expressed during early mammalian development. Genes Dev. 1989;3(6):848-859.

64. Ludewig P, et al. Carcinoembryonic antigen-related cell adhesion molecule 1 inhibits MMP-9-mediated blood-brain-barrier breakdown in a mouse model for ischemic stroke. Circ Res. 2013;113(8):1013-1022

65. Woodruff TM, et al. Species dependence for binding of small molecule agonist and antagonists to the C5a receptor on polymorphonuclear leukocytes. Inflammation. 2001;25(3):171-177. 